\title{
OPTIMAL-PARTITIONING INEQUALITIES FOR NONATOMIC PROBABILITY MEASURES
}

\author{
JOHN ELTON, THEODORE P. HILL AND ROBERT P. KERTZ ${ }^{1}$
}

\begin{abstract}
Suppose $\mu_{1}, \ldots, \mu_{n}$ are nonatomic probability measures on the same measurable space $(S, B)$. Then there exists a measurable partition $\left\{S_{i}\right\}_{i=1}^{n}$ of $S$ such that $\mu_{i}\left(S_{i}\right) \geq(n+1-M)^{-1}$ for all $i=1, \ldots, n$, where $M$ is the total mass of $\bigvee_{i=1}^{n} \mu_{i}$ (the smallest measure majorizing each $\mu_{i}$ ). This inequality is the best possibie for the functional $M$, and sharpens and quantifies a well-known cake-cutting theorem of Urbanik and of Dubins and Spanier. Applications are made to $L_{1}$-functions, discrete allocation problems, statistical decision theory, and a dual problem.
\end{abstract}

1. Introduction. Suppose $\mu_{1}, \ldots, \mu_{n}$ are nonatomic probability measures on the same measurable space $(S, B)$, and let $\Pi_{S}$ denote the collection of measurable partitions of $S$. Define

$$
\begin{aligned}
& M=M\left(\mu_{1}, \ldots, \mu_{n}\right)=\sup \left\{\sum_{i=1}^{n} \mu_{i}\left(A_{i}\right):\left\{A_{i}\right\}_{i=1}^{n} \in \Pi_{S}\right\} ; \text { and } \\
& v^{*}=v^{*}\left(\mu_{1}, \ldots, \mu_{n}\right)=\sup \left\{\min _{1 \leq i \leq n} \mu_{i}\left(A_{i}\right):\left\{A_{i}\right\}_{i=1}^{n} \in \Pi_{S}\right\} .
\end{aligned}
$$

The following is the main theorem in this paper (the upper bound in the conclusion is easy).

THEOREM 1.1. If $\mu_{1}, \ldots, \mu_{n}$ are nonatomic probability measures on $(S, B)$, then

$$
(n-M+1)^{-1} \leq v^{*} \leq M n^{-1},
$$

and these bounds are the best possible for the functional $M$.

In an $L_{1}$ framework, the framework in which the proof will be given, Theorem 1.1 (taking $f_{i} d \lambda=d \mu_{i}$ ) yields the following result.

THEOREM 1.2. Suppose $f_{1}, \ldots, f_{n}$ are nonnegative functions on a nonatomic finite measure algebra $(S, B, \lambda)$ and $\int_{S} f_{i} d \lambda=1$ for all $i=1, \ldots, n$. Then there exists a measurable partition $\left\{S_{i}\right\}_{i=1}^{n}$ of $S$ such that

$$
\int_{S_{i}} f_{i} d \lambda \geq(n-M+1)^{-1} \text { for all } i=1, \ldots, n,
$$

where $M=\int_{S} \max _{1 \leq i \leq n} f_{i} d \lambda$.

Received by the editors February 6, 1985 .

1980 Mathematics Subject Classification. Primary 60E15, 28A99; Secondary 90A05, 62C20, $60 \mathrm{~A} 10$.

Key words and phrases. Optimal-partitioning inequalities, cake-cutting, discrete allocation problems, minimax decision rules.

${ }_{1}$ John Elton was supported in part by National Science Foundation grant DMS-83-16627; Theodore P: Hill and Robert P. Kertz were supported in part by National Science Foundation grant DMS-84-01604. 
A cake-cutting interpretation of Theorem 1.1 based on a description by Dubins and Spanier in [4] is as follows. Suppose a cake $S$ is to be divided among $n$ people whose values $\left\{\mu_{i}\right\}_{i=1}^{n}$ of different portions of the cake may differ (here $\mu_{i}(A)$ represents the value of piece $A$ to person $i$ ). In [4] it was shown that if not all the values $\left\{\mu_{i}\right\}$ are identical, there are always a partition of $S$ into $n$ pieces so that each person receives a piece he values strictly more than $1 / n$. (This was apparently first proved by Urbanik [9] under the slightly less general assumption of mutual absolute continuity of the measures.)

COROLlaRY 1.3 (DUBINS AND SPANIER [4]). If $\mu_{1}, \ldots, \mu_{n}$ are nonatomic probability measures on $(S, B)$ and if $\mu_{i} \neq u_{j}$ for some $i \neq j$, then $v^{*}>1 / n$.

Proof. $M\left(\mu_{1}, \ldots, \mu_{n}\right)>1$ if and only if $\mu_{i} \neq \mu_{j}$ for some $i \neq j$.

In Theorem 1.1, $M$ may be viewed as the "cooperative" value of the cake to the $n$ people: the cake is cut optimally, each piece is given to a person who values it most, and the values of these pieces to the respective recipients are then pooled. On the other hand, $v^{*}$ represents the "noncooperative" (upper) value of the cake: the highest possible value any partition may assign to the person who gets the least. Thus Theorem 1.1 says that the noncooperative value always lies between one $n$th the cooperative value, $M$, and $(n-M+1)^{-1}$. (Observe that in the two extreme cases where the $\left\{\mu_{i}\right\}$ are identical and where the $\left\{\mu_{i}\right\}$ have disjoint supports, both bounds coincide, and are $n^{-1}$ and 1 respectively.)

Theorem 1.1 follows easily from Theorem 1.2. Theorem 1.2 is proved in a constructive approach through reduction and induction arguments. An averaging principle (Theorem 2.2) based on conditional expectations allows a reduction to special simple functions. These, in turn, are replaced by other simple functions which coincide on key maximizing sets, to which an induction (on $n$ ) is applied to obtain an essential partitioning inequality $(\S 3)$. $\S 4$ completes the proof of Theorem 1.2, and identifies the extremal distributions attaining the upper and lower bounds. $\S \S 5$ and 6 contain applications of these results to discrete allocation problems, and to a dual problem and statistical decision theory.

2. Optimal partitions and an averaging principle. The proofs of the main results in this paper, contained in this and the following two sections, will be given in the $L_{1}$ framework (that is, the setting of Theorem 1.2). In this section are proved the existence of optimal partitions (Theorem 2.1), an averaging principle (Theorem 2.2), and two reduction results (Propositions 2.3 and 2.4).

The following notation will be used throughout the paper: $(S, B, \lambda)$ is a nonatomic finite positive measure space; $\mathcal{F}_{S}$ is the set of nonnegative $B$-measurable functions $f$ with $\int_{S} f d \lambda=1$, and $\mathcal{F}_{S}^{n}=\mathcal{F}_{S} \times \cdots \times \mathcal{F}_{S}$ ( $n$ factors); $\bigvee_{i=1}^{n} a_{i}=$ $\max \left\{a_{i}: 1 \leq i \leq n\right\} ; A^{\prime}=S \backslash A$ is the complement of the set $A$ in $S ; 1_{A}$ is the indicator function of $A ; E(f \mid A)$ is the conditional expectation of $f$ given a sub- $\sigma$ algebra $A$ of $B ;\left.f\right|_{A}$ is the restriction of $f \in \mathcal{F}_{S}$ to the subset $A$ of $S ; P_{S}$ is the set of partitions of unity over $S$; that is, $P_{S}=\left\{\left\{\phi_{i}\right\}_{i=1}^{n}: \phi_{i} \in L_{\infty}(S), \phi_{i} \geq 0\right.$ for $i=1, \ldots, n$, and $\left.\sum_{i=1}^{n} \phi_{i} \leq 1\right\}$; and $\# K$ is the cardinality of the set $K$.

The $L_{1}$-analogs of (1.1) and (1.2) are

$$
M=M\left(f_{1}, \ldots, f_{n} ; \lambda\right)=\int_{S} \bigvee_{i=1}^{n} f_{i} d \lambda
$$


and

$$
\begin{aligned}
v^{*} & =v^{*}\left(f_{1}, \ldots, f_{n} ; \lambda\right) \\
& =\sup \left\{\min _{1 \leq i \leq n} \int_{A_{i}} f_{i} d \lambda:\left\{A_{i}\right\}_{i=1}^{n} \in \Pi_{S}\right\} .
\end{aligned}
$$

The first part of the next theorem, which guarantees the existence of optimal partitions, appears in Dubins and Spanier [4]; the proof given here is a simpler argument based on an idea of Dor [3].

THEOREM 2.1. Suppose $\left\{f_{i}\right\}_{i=1}^{n} \in \mathcal{F}_{S}^{n}$. Then there exists a measurable partition $\left\{S_{i}\right\}_{i=1}^{n}$ of $S$ satisfying

$$
\int_{S_{i}} f_{i} d \lambda \geq v^{*} \text { for all } i=1, \ldots, n
$$

Moreover,

$$
v^{*}=\sup \left\{\min _{1 \leq i \leq n} \int_{S} f_{i} \phi_{i} d \lambda:\left\{\phi_{i}\right\}_{i=1}^{n} \in P_{S}\right\}
$$

PrOOF. Let

$$
c^{*}=\sup \left\{\min _{1 \leq i \leq n} \int_{S} f_{i} \phi_{i} d \lambda:\left\{\phi_{i}\right\}_{i=1}^{n} \in P_{S}\right\},
$$

and note that $\left\{1_{S_{i}}\right\}_{i=1}^{n} \in P_{S}$ if $\left\{S_{i}\right\}_{i=1}^{n} \in \Pi_{S}$, so $v^{*} \leq c^{*}$. Choose $c_{1}, c_{2}, \ldots$ with $c_{m} \nearrow c^{*}$ and $\left\{\phi_{i}^{m}\right\}_{i=1}^{n} \in P_{S}$ such that $\min _{1 \leq i \leq n} \int_{S} f_{i} \phi_{i}^{m} d \lambda \geq c_{m}$. Let $B$ denote the unit ball of $L_{\infty}(S)$, the dual of $L_{1}(S)$, so $B$ is compact and metrizable in the $\mathrm{w}^{*}$-topology. By passing to subsequences, it may be assumed that $\left\{\phi_{i}^{m}\right\}_{m=1,2, \ldots}$ has a $w^{*}$-limit, call it $\phi_{i}$, as $m \rightarrow \infty$, for each $i=1, \ldots, n$. Clearly $\left\{\phi_{i}\right\}_{i=1}^{n} \in \mathcal{P}_{S}$ (just observe that $1-\phi_{i}^{m} \in B$ and $\sum_{i=1}^{n} \phi_{i}^{m} \in B$ for each $m=1,2, \ldots$, so the same is true for $\left.\left\{\phi_{i}\right\}_{i=1}^{n}\right)$. Also, $\int_{S} f_{i} \phi_{i}^{m} d \lambda \rightarrow \int_{S} f_{i} \phi_{i} d \lambda$ as $m \rightarrow \infty$ (because of the $\mathrm{w}^{*}$-convergence), so $\min _{1 \leq i \leq n} \int_{S} f_{i} \phi_{i} d \lambda \geq c^{*}$, and $c^{*}$ is attained by $\left\{\phi_{i}\right\}_{i=1}^{n} \in P_{S}$. Let $d_{i}=\int_{S} f_{i} \phi_{i} d \lambda$, so $d_{i} \geq c^{*}$ for $i=1, \ldots, n$.

To show $c^{*}=v^{*}$ and to obtain an optimal set partition, let

$$
K=\left\{\left\{\phi_{i}\right\}_{i=1}^{n} \in \mathcal{P}_{S}: \int_{S} f_{i} \phi_{i} d \lambda=d_{i}, i=1, \ldots, n\right\} .
$$

The set $K$ is a $\mathrm{w}^{*}$-closed, nonempty convex subset of the compact subset $B \times$ $B \times \cdots \times B$ of $L_{\infty}(S) \times \cdots \times L_{\infty}(S)$ with product topology of the w*-topology on each factor, a locally convex space; so the Kreĭn-Milman Theorem guarantees an extreme point $\left\{\psi_{i}\right\}_{i=1}^{n}$ of $K$. But it is easy to see that if $\lambda\left\{0<\psi_{i}<1\right\}>0$ for some $i=1, \ldots, n$, then $\left\{\psi_{i}\right\}_{i=1}^{n}$ could not be extreme. Hence there exist measurable subsets $\left\{S_{i}\right\}_{i=1}^{n}$ of $S$ such that $\psi_{i}=1_{S_{i}}$ for $i=1, \ldots, n$, and the $S_{i}$ 's must be pairwise disjoint (a.e.) since $\sum_{i=1}^{n} \psi_{i} \leq 1$. Clearly $\left\{S_{i}\right\}$ may be taken to be a partition of $S$, which completes the proof.

(Theorem 2.1 can be strengthened as follows. Define $v_{1}^{*}=v^{*}=v^{*}\left(f_{1}, \ldots, f_{n} ; \lambda\right)$, and for $k=2, \ldots, n$,

$$
\begin{aligned}
v_{k}^{*}=v_{k}^{*}\left(f_{1}, \ldots, f_{n} ; \lambda\right)=\sup \{c \geq & v_{k-1}^{*}: \exists\left\{S_{i}\right\}_{i=1}^{n} \in \Pi_{S} \\
& \text { with } \left.\left(\int_{S_{i}} f_{i} d \lambda\right)_{i=1}^{n} \geq\left(v_{1}^{*}, \ldots, v_{k-1}^{*}, c, \ldots, c\right)\right\},
\end{aligned}
$$


where $\left(a_{i}\right)_{i=1}^{n} \geq\left(b_{i}\right)_{i=1}^{n}$ means $a_{i}^{*} \geq b_{i}^{*}, i=1, \ldots, n$, for the nondecreasing rearrangements $\left(a_{i}^{*}\right)_{i=1}^{n}$ and $\left(b_{i}^{*}\right)_{i=1}^{n}$ of $\left(a_{i}\right)_{i=1}^{n}$ and $\left(b_{i}\right)_{i=1}^{n}$ respectively. Then the above arguments can be used to show there exists $\left\{S_{i}\right\}_{i=1}^{n} \in \Pi_{S}$ with $\left(\int_{S_{i}} f_{i} d \lambda\right)_{i=1}^{n} \geq$ $\left(v_{1}^{*}, v_{2}^{*}, \ldots, v_{n}^{*}\right)$, as was done by Dubins and Spanier [4] using a different approach.)

The next theorem provides one of the key tools to the proof of the main results (Theorems 1.1 and 1.2). Intuitively, it says that any averaging of the given functions (or measures) will decrease the "guaranteed minimum share" $v^{*}$. This is not surprising since fluctuations in functions generally allow "better" partitions simply by assigning to function $f_{i}$ intervals where $f_{i}$ is relatively larger than the other functions.

THEOREM 2.2. Let $\left\{f_{i}\right\}_{i=1}^{n} \in \mathcal{F}_{S}^{n}$ and let $\tilde{f}_{i}=E\left(f_{i} \mid \mathcal{A}\right)$ for $i=1, \ldots, n$, where $A$ is any sub- $\sigma$-algebra of $B$. Then $v^{*}\left(\tilde{f}_{1}, \ldots, \tilde{f}_{n} ; \lambda\right) \leq v^{*}\left(f_{1}, \ldots, f_{n} ; \lambda\right)$.

ProOF. First consider the case where $A$ is finite; that is, there is a partition $A_{1}, \ldots, A_{k}$ of $S$ so that each $A_{i}$ has positive measure and $A$ is the $\sigma$-algebra generated by $\left\{A_{1}, \ldots, A_{k}\right\}$. In this case,

$$
\tilde{f}_{i}=\sum_{j=1}^{k}\left(\lambda\left(A_{j}\right)^{-1} \int_{A_{j}} f_{i} d \lambda\right) 1_{A_{j}}, \quad i=1, \ldots, n .
$$

Let $\left\{S_{i}\right\}_{i=1}^{n} \in \Pi_{S}$; then from (2.3) it follows that

$$
\int_{S_{i}} \tilde{f}_{i} d \lambda=\sum_{j=1}^{k} \int_{S_{i} \cap A_{j}} \tilde{f}_{i} d \lambda=\sum_{j=1}^{k} \lambda\left(S_{i} \cap A_{j}\right) \lambda\left(A_{j}\right)^{-1} \int_{A_{j}} f_{i} d \lambda .
$$

Let

$$
\phi_{i}=\sum_{j=1}^{k} \lambda\left(S_{i} \cap A_{j}\right) \lambda\left(A_{j}\right)^{-1} \cdot 1_{A_{j}} \quad \text { for } i=1, \ldots, n ;
$$

then $\sum_{i=1}^{n} \phi_{i}=1$, so $\left\{\phi_{i}\right\}_{i=1}^{n} \in P_{S}$. From (2.4),

$$
\int_{S} f_{i} \phi_{i} d \lambda=\sum_{j=1}^{k} \int_{A_{j}} f_{i} \phi_{i} d \lambda=\int_{S_{i}} \tilde{f}_{i} d \lambda
$$

It now follows from Theorem 2.1 that there exists a partition $\left\{B_{i}\right\}_{i=1}^{n} \in \Pi_{S}$ such that $\min _{i} \int_{B_{i}} f_{i} d \lambda \geq \min _{i} \int_{S_{i}} \tilde{f}_{i} d \lambda$. Thus, the conclusion of Theorem 2.2 holds for this case.

In the general case, we may clearly assume without loss of generality that $B$ is complete and separable (by taking the completion of the sub- $\sigma$-algebra generated by the $f_{i}$ 's), and that $A$ is a complete, separable subalgebra of $B$ (use the completion if necessary; separability here means that the measure subalgebra of $A$ modulo $\lambda$ null sets is a separable metric space). It follows that there exist increasing finite subalgebras $A_{1}, A_{2}, \ldots$ of $A$ such that $A$ is generated by $\bigcup_{m=1}^{\infty} A_{m}$. Let $\tilde{f}_{i}^{m}=$ $E\left(f_{i} \mid A_{m}\right)$ for $m=1,2, \ldots, i=1, \ldots, n$. It then follows from the finite case that

$$
\min _{1 \leq i \leq n} \int_{S_{i}} \tilde{f}_{i}^{m} d \lambda \leq v^{*}\left(f_{1}, \ldots, f_{n} ; \lambda\right) \quad \text { for each } m=1,2, \ldots
$$


Since $\tilde{f}_{i}^{m} \rightarrow \tilde{f}_{i}$ (in $\left.L^{1}(S)\right)$ as $m \rightarrow \infty$, by Levy's martingale convergence theorem (e.g., [1, p. 298]),

$$
\min _{1 \leq i \leq n} \int_{S_{i}} \tilde{f}_{i} d \lambda \leq v^{*}\left(f_{1}, \ldots, f_{n} ; \lambda\right)
$$

also.

The next two propositions allow restrictions to special subsets of $\exists_{S}^{n}$ in the proof of Theorem 1.2.

PROPOSITION 2.3. If the conclusion of Theorem 1.2 holds for all systems $\left\{f_{i}\right\}_{i=1}^{n} \in \mathcal{F}_{S}^{n}$ which satisfy, in addition,

$$
\lambda\left\{f_{k}>\bigvee_{i \neq k} f_{i}\right\}>0 \text { for } k=1, \ldots, n,
$$

then it holds for all $\left\{f_{i}\right\}_{i=1}^{n} \in \mathcal{F}_{S}^{n}$.

ProOF. Fix $\varepsilon>0$. Using the fact that $\lambda$ is nonatomic, it is easy to find $n$ disjoint subsets of $S$ of small measure, with $f_{i}>0$ on the $i$ th subset, and redefine the $f_{i}$ 's on these sets so that the modified functions $\left\{\hat{f}_{i}\right\}_{i=1}^{n}$ satisfy (2.5), are in $\mathcal{F}_{S}, M\left(\hat{f}_{1}, \ldots, \hat{f}_{n} ; \lambda\right) \geq M\left(f_{1}, \ldots, f_{n} ; \lambda\right)$, and $\left|\int_{S_{i}} \hat{f}_{i} d \lambda-\int_{S_{i}} f_{i} d \lambda\right|<\varepsilon$ for all $\left\{S_{i}\right\}_{i=1}^{n} \in \Pi_{S}$. The conclusion then follows from the hypothesis and the existence of optimal partitions for $\left\{f_{i}\right\}_{i=1}^{n}$ (Theorem 2.1).

The sets where each function $f_{i}$ majorizes the other functions play a special role in the next section.

DEFINITION 2.4. For $\left\{f_{i}\right\}_{i=1}^{n} \in \mathcal{F}_{S}^{n},\left\{A_{i}\right\}_{i=1}^{n}=\left\{A_{i}\left(f_{1}, \ldots, f_{n}\right)\right\}_{i=1}^{n} \in \Pi_{S}$ is given by $A_{1}=\left\{f_{1}=\bigvee_{i=1}^{n} f_{i}\right\}$, and $A_{k}=\left\{f_{k}=\bigvee_{j=1}^{n} f_{j}\right\} \backslash \bigcup_{j=1}^{k-1} A_{j}$ for $k=$ $2, \ldots, n$; and $A\left(f_{1}, \ldots, f_{n}\right)$ is the subalgebra of $B$ generated by $\left\{A_{i}\right\}_{i=1}^{n}$.

Proposition 2.5. Let $\left\{f_{i}\right\}_{i=1}^{n} \in \mathcal{F}_{S}^{n}$, and define $\left\{\tilde{f}_{i}\right\}_{i=1}^{n} \in \mathcal{F}_{S}^{n}$ by $\tilde{f}_{i}=$ $E\left(f_{i} \mid \mathcal{A}\right)$, where $A=A\left(f_{1}, \ldots, f_{n}\right)$. Then if the conclusion of Theorem 1.2 holds for $\left\{\tilde{f}_{i}\right\}_{i=1}^{n}$, it holds for $\left\{f_{i}\right\}_{i=1}^{n}$.

ProOF. Observe that $\int_{S} \bigvee_{i=1}^{n} \tilde{f}_{i} d \lambda=\int_{S} \bigvee_{i=1}^{n} f_{i} d \lambda=M$, and apply the hypothesis and Theorem 2.2 to conclude that $(n+1-M)^{-1} \leq v^{*}\left(\tilde{f}_{1}, \ldots, \tilde{f}_{n} ; \lambda\right) \leq$ $v^{*}\left(f_{1}, \ldots, f_{n} ; \lambda\right)$ and Theorem 2.1 to conclude the existence of optimal partitions for $\left\{f_{i}\right\}_{i=1}^{n}$.

3. Two key propositions. The results of this section form the core of the proof of Theorem 1.1 to be given in $\S 4$; recall that the proofs will be given in the $L_{1}$-setting of Theorem 1.2.

For the remainder of this section, fix $n>1$ and make the following induction assumption.

(3.1) INDUCTION ASSUMPTION. Theorem 1.2 holds for all $\tilde{n}=1,2, \ldots, n-1$.

Also for the remainder of this section, fix $\left\{f_{i}\right\}_{i=1}^{n} \in \mathcal{F}_{S}^{n}$, let $M$ be as in (2.1), and let $\left\{A_{i}\right\}_{i=1}^{n} \in \Pi_{S}$ be as in Definition 2.4. By Propositions 2.3 and 2.5 it may further be assumed (in order to prove Theorem 1.2) that

(3.2) $\left.f_{i}\right|_{A_{j}}$ is constant for each $i, j=1, \ldots, n$; and

(3.3) $\int_{A_{k}} f_{k} d \lambda>0$ for $k=1, \ldots, n$. 
By renumbering if necessary, there exists a $1 \leq m \leq n$ so that

$$
\int_{A_{i}} f_{i} d \lambda \geq(n+1-M)^{-1} \quad \text { if and only if } i \leq m \text {. }
$$

(To see (3.4), observe that $(n+1-M)^{-1} \leq M n^{-1}$ since $1 \leq M \leq n$ and that for some $i \leq n, \int_{A_{i}} f_{i} d \lambda \geq M n^{-1}$.)

If $m=n$, then the conclusion of Theorem 1.2 holds for $\left\{f_{i}\right\}_{i=1}^{n}$, so assume $m<n$.

The following notation is also used in this and the next section:

(i) $\left\{S_{i}\right\}_{i=1}^{m}$ satisfy $S_{i} \subseteq A_{i}$, and $\int_{S_{i}} f_{i} d \lambda=(n+1-M)^{-1}$ for $i=1, \ldots, m$;

(ii) $Q=S \backslash \bigcup_{i=1}^{m} S_{i}$;

(iii) $\left\{T_{i}\right\}_{i=1}^{m}$ are given by $T_{i}=A_{i} \backslash S_{i}$;

(iv) $\mathcal{G}_{S}=\left\{g \in \mathcal{F}_{S}:\left.g\right|_{S_{i}},\left.g\right|_{T_{i}}\right.$ and $\left.g\right|_{A_{k}}$ are constant for $i=1, \ldots, m, k=m+$ $1, \ldots, n\}$;

and for $J \subseteq K \subseteq\{m+1, \ldots, n\}$,

(v) $\mathcal{M}(J, K)=\left\{\left\{g_{k}\right\}_{k \in K} \subset \mathcal{G}_{S}\right.$ satisfying (3.5) and (3.7) below $\}$;

(vi) $\mathcal{M}(K)=\mathcal{M}(K, K)$; and

(vii) $\mathcal{N}(J, K)=\left\{\left\{g_{k}\right\}_{k \in K} \subset \mathcal{G}_{S}\right.$ satisfying (3.6) and (3.7) below $\}$.

(3.5) For each $i \in\{1, \ldots, m\}, \exists j_{i} \in J$ and $u_{i} \geq 0$ with

$$
\left.g_{j_{i}}\right|_{A_{i}}=u_{i}, \text { and }\left.g_{j}\right|_{S_{i}}=u_{i} \geq\left. g_{j}\right|_{T_{i}} \quad \text { for all } j \in J \text {. }
$$

(3.6) There exist $j_{i} \in J$ and $u_{i} \geq 0$ for $i=1, \ldots, m$, satisfying

$$
\begin{gathered}
\left.g_{j_{i}}\right|_{A_{i}}=u_{i},\left.g_{j_{k}}\right|_{S_{i}}=u_{i} \text { for all } k=1, \ldots, m, \text { and } \\
\left.g_{j}\right|_{A_{i}} \leq u_{i} \text { for all } j \in J, \text { for each } i=1, \ldots, m .
\end{gathered}
$$

(3.7) For every $k \in K,\left.g_{k}\right|_{A_{k}}=\left.f_{k}\right|_{A_{k}}$ and $g_{k} \leq \bigvee_{i=1}^{n} f_{i}$.

REMARKS. The existence of the $\left\{S_{i}\right\}_{i=1}^{m}$ follows from (3.4) and the fact that $\lambda$ is nonatomic. (As will be seen later in the proof, these disjoint sets will form the first $m$ elements of the partition sought in the conclusion of Theorem 1.2.) For systems $\left\{g_{k}\right\}_{k \in K} \in \mathcal{M}(J, K)$, the subcollection $\left\{g_{j}: j \in J\right\}$ are identical (with constant value $u_{i}$ ) on $S_{i}$, and the maximum of this subcollection on $T_{i}$ also has value $u_{i}$ for each $i=1, \ldots, m$ (see Figure I). On the other hand, for $\left\{g_{k}\right\}_{k \in K} \in \mathcal{N}(J, K)$, only a subset of $\left\{g_{j}: j \in J\right\}$ coincide on $\bigcup_{i=1}^{m} S_{i}$; hence $\mathcal{M}(J, K) \subseteq \mathcal{N}(J, K)$ (see Figures I and $\mathrm{I}^{\prime}$ ).

PROPOSITION 3.3. If $\left\{g_{j}\right\}_{j=m+1}^{n} \in \mathcal{M}(\{m+1, \ldots, n\})$, there exist $\left\{S_{j}\right\}_{j=m+1}^{n}$ $\in \Pi_{Q}$ such that

$$
\int_{S_{j}} g_{j} d \lambda \geq(n+1-M)^{-1} \text { for each } j=m+1, \ldots, n .
$$

PROOF. Suppose $\left\{g_{j}\right\}_{j=m+1}^{n} \in \mathcal{M}(\{m+1, \ldots, n\})$. From (3.5) the functions $\left\{g_{j}\right\}_{j=m+1}^{n}$ all have the same integral over $Q$, namely,

$$
\int_{Q} g_{j} d \lambda=1-\int_{S \backslash Q} g_{j} d \lambda=1-\sum_{i=1}^{m} u_{i} \lambda\left(S_{i}\right)=: c
$$


where $c>0$ since $\int_{Q} g_{j} d \lambda \geq \int_{A_{j}} g_{j} d \lambda=\int_{A_{j}} f_{j} d \lambda$, which is $>0$ by (3.3). Define $\bar{g}_{j}=\left.c^{-1} g_{j}\right|_{Q}$, so $\int_{Q} \bar{g}_{j} d \lambda=1$, by $(3.9)$, for each $j=m+1, \ldots, n$. Let $\bar{M}=$ $\int_{Q} \bigvee_{j=m+1}^{n} \bar{g}_{j} d \lambda ;$ then

$$
\bar{M}=c^{-1}\left[\sum_{j=m+1}^{n} \int_{A_{j}} f_{j} d \lambda+\sum_{j=1}^{m} u_{j} \lambda\left(T_{j}\right)\right]
$$

by the definition of $\mathcal{M}(\{m+1, \ldots, n\})$. By the induction assumption (3.1) applied to $\left\{\bar{g}_{j}\right\}_{j=m+1}^{n} \in \mathcal{F}_{Q}^{n-m}$, there exists $\left\{S_{j}\right\}_{j=m+1}^{n} \in \Pi_{Q}$ such that $\int_{S_{j}} \bar{g}_{j} d \lambda \geq$ $(n-m+1-\bar{M})^{-1}$, so $\int_{S_{j}} g_{j} d \lambda \geq c(n-m+1-\bar{M})^{-1}$, for each $j=m+1, \ldots, n$.

To complete the proof of the proposition, it is enough to show

$$
c(n-m+1-\bar{M})^{-1} \geq(n+1-M)^{-1},
$$

which is equivalent to showing

$$
\bar{M} \geq M-m+(n+1-M) \sum_{i=1}^{m} u_{i} \lambda\left(S_{i}\right) .
$$

Let $w_{i}=$ constant $=\left.f_{i}\right|_{A_{i}}$ for $i=1, \ldots, m$. Note that $w_{i} \geq u_{i}$ (from (3.7)); $w_{i} \lambda\left(S_{i}\right)=(n+1-M)^{-1}$ (by definition of $S_{i}$ ); and $w_{i} \lambda\left(A_{i}\right)=\int_{A_{i}} f_{i} d \lambda \leq 1$ for each $i=1, \ldots, m$. This implies

$$
\begin{aligned}
M & -m+(n+1-M) \sum_{i=1}^{m} u_{i} \lambda\left(S_{i}\right)=M-\sum_{i=1}^{m}\left(1-u_{i} w_{i}^{-1}\right) \\
& \leq M-\sum_{i=1}^{m} \lambda\left(A_{i}\right) w_{i}\left(1-u_{i} w_{i}^{-1}\right)=M-\sum_{i=1}^{m} \int_{A_{i}} f_{i} d \lambda+\sum_{i=1}^{m} u_{i} \lambda\left(A_{i}\right) \\
& =\sum_{i=m+1}^{n} \int_{A_{i}} f_{i} d \lambda+\sum_{i=1}^{m} u_{i} \lambda\left(A_{i}\right)
\end{aligned}
$$

since $M=\sum_{i=1}^{n} \int_{A_{i}} f_{i} d \lambda$ by the definition of $\left\{A_{i}\right\}_{i=1}^{n}$. Also,

$$
\sum_{i=m+1}^{n} \int_{A_{i}} f_{i} d \lambda+\sum_{i=1}^{m} u_{i} \lambda\left(A_{i}\right) \geq 1
$$

since $\sum_{i=1}^{m} u_{i} 1_{A_{i}}+\sum_{i=m+1}^{n} f_{i} 1_{A_{i}}$ dominates $g_{j}$ for any $j=m+1, \ldots, n$. Using the definition (3.9) of $c$ and noting that $0<c \leq 1$, it follows that

$$
\begin{aligned}
& \sum_{i=m+1}^{n} \int_{A_{i}} f_{i} d \lambda+\sum_{i=1}^{m} u_{i} \lambda\left(A_{i}\right) \\
& \quad \leq c^{-1}\left(\sum_{i=m+1}^{n} \int_{A_{i}} f_{i} d \lambda+\sum_{i=1}^{m} u_{i}\left(\lambda\left(A_{i}\right)-\lambda\left(S_{i}\right)\right)\right)=\bar{M}
\end{aligned}
$$

and (3.10) follows from (3.11) and (3.12).

The next lemma allows, for certain special classes of functions, a reduction to the case considered by Proposition 3.3. 


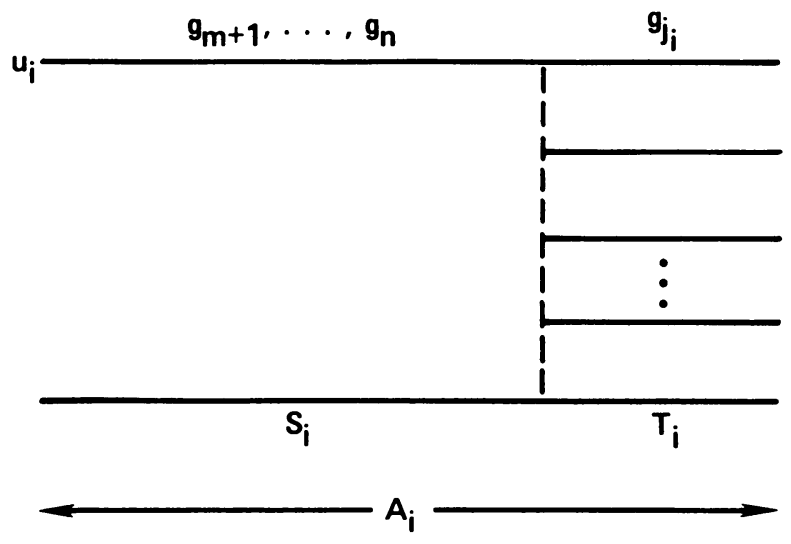

FIGURE I. Representations of $\left\{g_{j}\right\}_{j=m+1}^{n}$ in $\mathcal{M}(\{m+1, \ldots, n\})$ over set $A_{i}$, for $i=1, \ldots, m$.

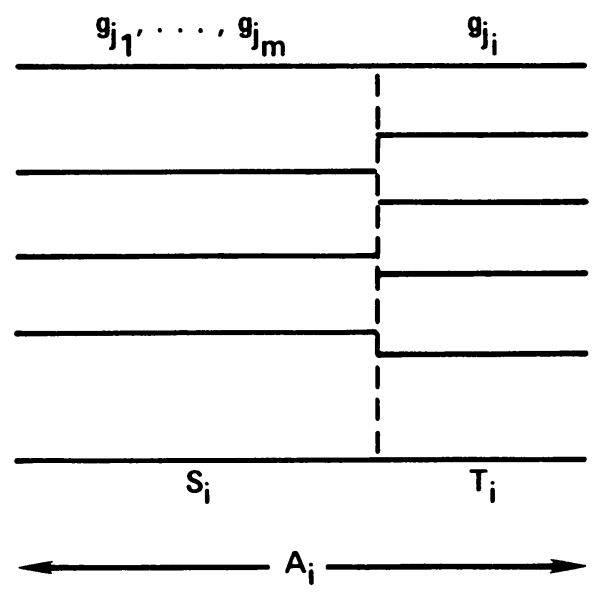

FigURE I'. Representation of $\left\{g_{j}\right\}_{j=m+1}^{n}$ in $\mathcal{N}(\{m+1, \ldots, n\},\{m+1, \ldots, n\})$ over set $A_{i}$, for $i=1, \ldots, m$.

LEMMA 3.4. Let $J$ be a subset of $\{m+1, \ldots, n\}$, and let $\left\{h_{j}\right\}_{j=m+1}^{n}$ be in $\mathcal{N}(J,\{m+1, \ldots, n\})$. Then there exist $\left\{g_{j}\right\}_{j=m+1}^{n}$ in $\mathcal{M}(J,\{m+1, \ldots, n\})$ satisfying

$$
\begin{aligned}
& \left.g_{j}\right|_{Q} \leq\left. h_{j}\right|_{Q} \text { for } j \in J \backslash\left\{j_{1}, \ldots, j_{m}\right\}, \text { and } \\
& g_{j}=h_{j} \text { for } j \notin J \backslash\left\{j_{1}, \ldots, j_{m}\right\}, \\
& \text { where } j_{1}, \ldots, j_{m} \text { are as in }(3.6) \text { for }\left\{h_{j}\right\}_{j=m+1}^{n} .
\end{aligned}
$$

PROOF. Let $\left\{h_{j}\right\}_{j=m+1}^{n}$ satisfy the hypothesis, and $j_{1}, \ldots, j_{m}$ be as in (3.6). For $j \in J \backslash\left\{j_{1}, \ldots, j_{m}\right\}$, increase $h_{j}$ to $u_{1}$ on $S_{1}$, then to $u_{2}$ on $S_{2}, \ldots, u_{m}$ on $S_{m}$, keeping $h_{j}$ normalized by decreasing it on $\left(\bigcup_{i=1}^{m} S_{i} \cup A_{j}\right)^{\prime}$ but not below zero (this will also preserve $\left.h_{j}\right|_{A_{j}}=\left.f_{j}\right|_{A_{j}}$ ). For simplicity of notation, in this proof the modified functions will still be denoted $\left\{h_{j}\right\}$. All modifications can be done in such a way as to remain in $\mathcal{G}_{S}$. It will be shown below that, in fact, $h_{j}$ does increase to $u_{i}$ on $S_{i}$ for every $i=1, \ldots, m$, and every $j \in J \backslash\left\{j_{1}, \ldots, j_{m}\right\}$. Then set $g_{j}=$ "new" $h_{j}$ 
for each $j \in J \backslash\left\{j_{1}, \ldots, j_{m}\right\}$, and = "original" $h_{j}$ for $j \notin J \backslash\left\{j_{1}, \ldots, j_{m}\right\}$; these $\left\{g_{j}\right\}$ satisfy the conclusion of the lemma.

It will now be shown that the above always happens; for suppose that for at least one $\hat{j} \in J \backslash\left\{j_{1}, \ldots, j_{m}\right\}, h_{\hat{j}}$ becomes zero on $\left(\bigcup_{i=1}^{m} S_{i} \cup A_{\hat{j}}\right)^{\prime}$ before it reaches $u_{i}$ on each set $S_{i}, i=1, \ldots, m$. It shall now be shown that this leads to a contradiction.

First, for each $j \notin J, m+1 \leq j \leq n$, attempt to increase $h_{j}$ to $u_{i}$ on $S_{i}$ for those $i$ where $\left.h_{j}\right|_{A_{i}}<u_{i}$; at the same time, preserve normalization by decreasing $h_{j}$ on $\left(\bigcup_{i=1}^{m} S_{i} \cup A_{j}\right)^{\prime}$ but not below zero, and decreasing $h_{j}$ on $S_{i}$, but not below $u_{i}$, for those $i=1, \ldots, m$ for which $\left.h_{j}\right|_{S_{i}}>u_{i}$. A "new" function $h_{j}$ is obtained which satisfies either $\left.h_{j}\right|_{S_{i}} \geq u_{i}$ for each $i=1, \ldots, m$, or $h_{j}=0$ on $\left(\bigcup_{i=1}^{m} S_{i} \cup A_{j}\right)^{\prime}$ and $h_{j} \mid S_{i} \leq u_{i}$ for each $i=1, \ldots, m$.

Using the functions $\left\{h_{j}\right\}_{j=m+1}^{n}$ thus obtained, define a set of indices $Z$ by $Z=$ $\left\{j: m+1 \leq j \leq n\right.$, and $h_{j}=0$ on $\left(\bigcup_{i=1}^{m} S_{i} \cup A_{j}\right)^{\prime}$, while $\left.h_{j}\right|_{S_{i}} \leq u_{i}$ for each $i=1, \ldots, m\}$. Then $Z \neq \varnothing$, since $\hat{j} \in Z$.

Let $j_{0} \in Z$ be such that

$$
\int_{\bigcup_{i=1}^{m} S_{i}} h_{j_{0}} d \lambda=\min \left\{\int_{\bigcup_{i=1}^{m} S_{i}} h_{j} d \lambda: j \in Z\right\} .
$$

For each $j \in Z$ increase or decrease $h_{j}$ on each $S_{i}$ as necessary, keeping $h_{j}$ normalized (and nonnegative) so that $u_{i} \geq\left. h_{j}\right|_{S_{i}} \geq h_{j_{0}} \mid s_{i}$ for $i=1, \ldots, m$; and for $j \in\{m+1, \ldots, n\} \backslash Z,\left.h_{j}\right|_{S_{i}} \geq u_{i} \geq\left. h_{j_{0}}\right|_{S_{i}}$ for $i=1, \ldots, m$. Thus $\left.h_{j}\right|_{S_{i}} \geq\left. h_{j_{0}}\right|_{S_{i}}$ for all $i=1, \ldots, m$ and $j=m+1, \ldots, n$, and $h_{j_{0}}=0$ on $\left(\bigcup_{i=1}^{m} S_{i} \cup A_{j_{0}}\right)^{\prime}$. This implies each $h_{j}$ dominates $h_{j_{0}}$ everywhere except on $A_{j_{0}}$. Since both functions $h_{j}$ and $h_{j_{0}}$ have the same integral (namely 1 ) on $S$, it follows that $\left.h_{j}\right|_{S_{i}}$ may be decreased to agree with $\left.h_{j_{0}}\right|_{S_{i}}$ for each $i=1, \ldots, m$, keeping $h_{j}$ normalized by increasing $h_{j}$ on $A_{j_{0}} ;\left.h_{j}\right|_{A_{j_{0}}}$ will increase above $\left.h_{j_{0}}\right|_{A_{j_{0}}}=\left.f_{j}\right|_{A_{j_{0}}}$, for each $j=m+1, \ldots, n$. Also $\left.h_{j}\right|_{i}$ may be decreased to the number $\left.h_{j_{0}}\right|_{S_{i}}$ (if it was greater) in the same way. (This was the case for $\left.h_{j_{i}}\right|_{T_{i}}=u_{i}, i=1, \ldots, m$; the modification makes $\left.h_{j_{i}}\right|_{A_{i}}$ constant for the new function, for all $i=1, \ldots, m$.)

After these modifications, the new functions $\left\{h_{j}\right\}_{j=m+1}^{n}$ are in $\mathcal{M}(\{m+1, \ldots, n\})$, so by Proposition 3.3 there exists a set $S_{j_{0}} \subseteq Q$ such that

$$
\int_{S_{j_{0}}} h_{j_{0}} d \lambda \geq(n+1-M)^{-1}
$$

Since $h_{j_{0}}=0$ on $Q \backslash A_{j_{0}}$, this implies that $\int_{A_{j_{0}}} h_{j_{0}} d \lambda \geq(n+1-M)^{-1}$ and thus $\int_{A_{j_{0}}} f_{j_{0}} d \lambda \geq(n+1-M)^{-1}$ (since $\left.h_{j_{0}}\right|_{A_{j_{0}}}=\left.f_{j_{0}}\right|_{A_{j_{0}}}$ ), which contradicts the definition (3.4) of $m$, since $j_{0}>m$. Thus $Z$ must have been empty, which completes the proof.

(Note that in this proof the conclusion of Proposition 3.3 was applied to $h_{j_{0}}$ and not to the other $h_{j}$ 's, which were increased on $A_{j_{0}}$.)

The next proposition will be used in the proof of the main theorem in the next section; it shows that the functions $\left\{f_{i}\right\}_{i=1}^{n}$ can be "merged" on $S_{1}, \ldots, S_{m}$ by decreasing the value of $f_{j}$ on $\left(\bigcup_{i=1}^{m} S_{i} \cup A_{j}\right)^{\prime}$ in such a way as to satisfy the hypotheses of Proposition 3.3. 

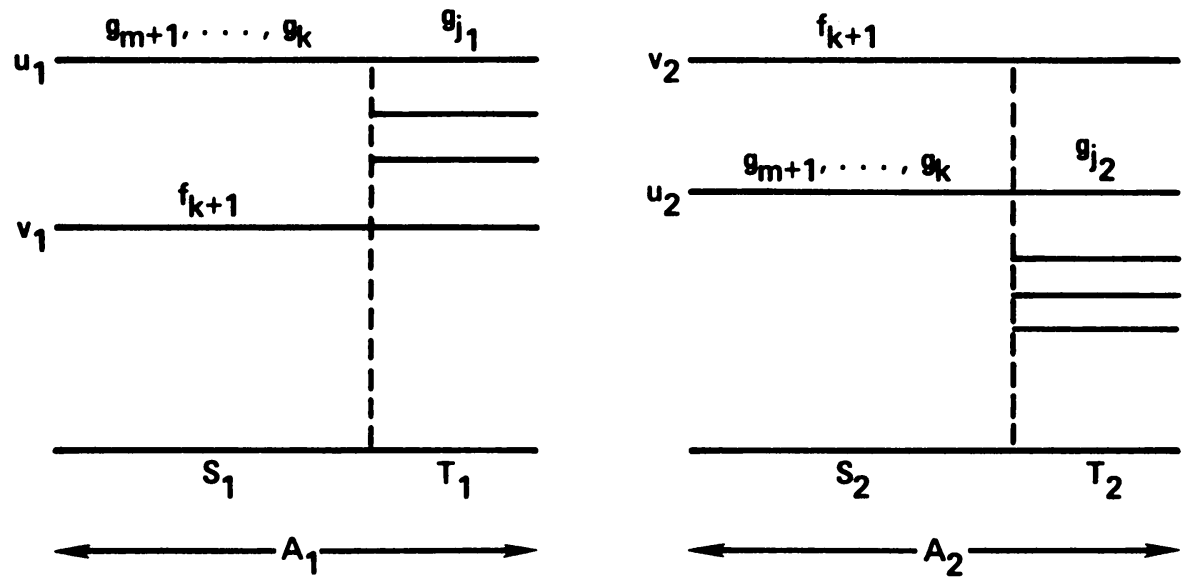

FIGURE II. Representation of $\left\{g_{j}\right\}_{j=m+1}^{k}$ and $f_{k+1}$ over $A_{1}$ and $A_{2}$, if $1 \in I$ and $2 \in I^{\prime}$, at the beginning of the proof of Proposition 3.5 .

Proposition 3.5. There exists $\left\{g_{j}\right\}_{j=m+1}^{n} \in \mathcal{M}(\{m+1, \ldots, n\})$ satisfying, for all $j=m+1, \ldots, n$,

$$
\left.g_{j}\right|_{Q} \leq\left. f_{j}\right|_{Q}
$$

The proof will be by induction. First, observe that (3.14) is satisfied for $j=$ $m+1, g_{m+1}=f_{m+1} \in \mathcal{M}(\{m+1\})$, and $j_{i}=m+1$ for all $i=1, \ldots, m$. The induction statement is then

(3.15) if there exists $\left\{g_{j}\right\}_{j=m+1}^{k} \in \mathcal{M}(\{m+1, \ldots, k\})$ which satisfies (3.14)

$$
\begin{aligned}
& \text { for } j=m+1, \ldots, k<n, \text { then there exists }\left\{\hat{g}_{j}\right\}_{j=m+1}^{k+1} \in \\
& \mathcal{M}(\{m+1, \ldots, k+1\}) \text { which satisfies }(3.14) \text { for } j=m+1, \ldots, k+1 .
\end{aligned}
$$

For the remainder of this section, fix $k, m<k<n$, and fix $\left\{g_{j}\right\}_{j=m+1}^{k}$ as in (3.15) with $j_{1}, \ldots, j_{m}$ as in (3.5).

(The hypothesis of (3.15) says that the $g_{j}$ 's, $j=m+1, \ldots, k$, coincide and are below $\bigvee_{i=1}^{n} f_{i}$ on each $S_{i}, i=1, \ldots, m$; that $g_{j}$ is below $f_{j}$ elsewhere and agrees with $f_{j}$ on $A_{j}$; and that the largest $g_{j}$ on $T_{i}$ is in fact constant on $A_{i}$ for $i=1, \ldots, m$. It shall now be shown how to "merge" $f_{k+1}$ with $g_{m+1}, \ldots, g_{k}$ on $\bigcup_{i=1}^{m} S_{i}$ by modifying $g_{m+1}, \ldots, g_{k}, f_{k+1}$ in such a way that they remain in $\mathcal{G}_{S}$, only decrease on $Q$, and the resulting collection satisfies (3.7).)

Let $\left\{u_{i}\right\}_{i=1}^{m}$ and $\left\{v_{i}\right\}_{i=1}^{m}$ be the constants

$$
u_{i}=\left.g_{j_{i}}\right|_{A_{i}} \text { and } \quad v_{i}=\left.f_{k+1}\right|_{A_{i}} \quad \text { for } i=1, \ldots, m .
$$

(These constants will remain fixed throughout the proof.) Note that $f_{k+1}$ is strictly above $u_{i}$ on some $A_{i}$ 's and below or equal $u_{i}$ on others, for $i=1, \ldots, m$. Partition the indices $\{1, \ldots, m\}$ into sets $I$ and $I^{\prime}$ by

$$
I=\left\{1 \leq i \leq m: v_{i} \leq u_{i}\right\} \text { and } I^{\prime}=\left\{1 \leq i \leq m: v_{i}>u_{i}\right\}
$$

(see Figure II).

If $I$ (respectively $I^{\prime}$ ) were empty, Lemma 3.4 could be applied with $J=\{m+$ $1, \ldots, k+1\}$ and $g_{m+1}, \ldots, g_{k}, f_{k+1}, \ldots, f_{n}$ in the role of $\left\{h_{j}\right\}_{j=m+1}^{n}$, and $k+1$ 

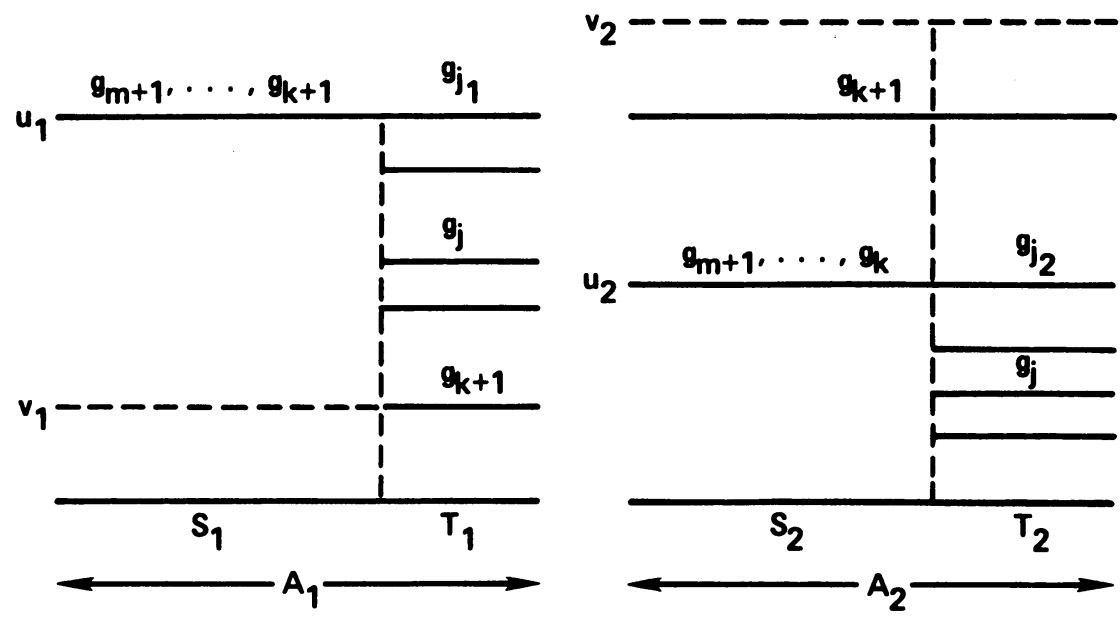

FIGURE III. Representation of $\left\{g_{j}\right\}_{j=m+1}^{k+1}$ over $A_{1}$ and $A_{2}$, if $1 \in I$ and $2 \in I^{\prime}$, when case (3.18) occurs in the proof of Proposition 3.5.

(respectively $\left.\left\{j_{1}, \ldots, j_{k}\right\}\right)$ in the role of the indices in (3.6), to complete the proof of (3.15). Therefore assume both $I \neq \varnothing$ and $I^{\prime} \neq \varnothing$.

Consider decreasing $f_{k+1}$ on $A_{i}$ (but not below $u_{i}$ ) for $i \in I^{\prime}$, and increasing $f_{k+1}$ on $S_{i}$ (but not above $u_{i}$ ) for $i \in I$, keeping $f_{k+1}$ normalized (not changing $f_{k+1}$ over $\left.\left(\bigcup_{i \in I} T_{i}\right) \cup\left(\bigcup_{i=m+1}^{n} A_{i}\right)\right)$. Let $g_{k+1}$ denote the function resulting from this modification of $f_{k+1}$ carried as far as possible. If $\left.g_{k+1}\right|_{A_{i}}=u_{i}$ for all $i \in I^{\prime}$ and $\left.g_{k+1}\right|_{S_{i}} \leq u_{i}$ (note $\left.g_{k+1}\right|_{T_{i}}=v_{i} \leq u_{i}$ for all $i \in I$ ), then since $\left.g_{k+1}\right|_{A_{i}} \leq u_{i}$ for all $i=1, \ldots, m$, Lemma 3.4 may be applied with $J=\{m+1, \ldots, k+1\}$ and $\left\{h_{j}\right\}_{j=m+1}^{n}=\left\{g_{m+1}, \ldots, g_{k+1}, f_{k+2}, \ldots, f_{n}\right\}$ to establish (3.15). (The $h_{j}$ 's satisfy $\left.h_{j}\right|_{Q} \leq\left. f_{j}\right|_{Q}$ for $j=m+1, \ldots, k+1$, and hence the functions $\left\{\hat{g}_{j}\right\}_{j=m+1}^{k+1}$ guaranteed by Lemma 3.4 satisfy (3.13), and thus also satisfy (3.14).) Therefore, it may instead be assumed that

$$
\begin{array}{ll}
\left.g_{k+1}\right|_{A_{i}} \geq u_{i} & \text { for all } i \in I^{\prime} \\
\left.g_{k+1}\right|_{S_{i}}=u_{i} & \text { for all } i \in I
\end{array}
$$

(see Figure III).

Informal description of the remainder of the proof of (3.15). The idea is to decrease together the functions over $S_{i}$ (and over $T_{i}$ as well for those functions which are the largest on $T_{i}$ ) for each $i \in I$, at a possibly different rate for each $i$, so that at all times a complete set of "representatives" of the largest functions on $T_{i}, i \in I$, will coincide on $S_{p}$ and will be the largest (among $\left\{g_{m+1}, \ldots, g_{k}\right\}$ ) functions on $S_{p}$ for $p \in I^{\prime}$. (Observe that decreasing the functions as described on $A_{i}, i \in I$, is balanced by increasing $g_{m+1}, \ldots, g_{k}$ on $S_{p}, p \in I^{\prime}$, in order to preserve normalization. Note also that as the functions decrease over $A_{i}$, more functions may be "picked up" as being among the largest on $T_{i}$.)

The functions $g_{k+1}$ must be increased somewhere to compensate for decreasing it on $S_{i}, i \in I$, so increase $g_{k+1}$ over all of $A_{p}, p \in I^{\prime}$, but not above $v_{p}$, the original value of $f_{k+1}$ on $A_{p}, p \in I^{\prime}$. Observe that for $i \in I, g_{k+1}$ decreases only on $S_{i}$, but some $g_{j}$ 's decrease on all of $A_{i}$. Also, for $p \in I^{\prime}, g_{k+1}$ must increase on all of 

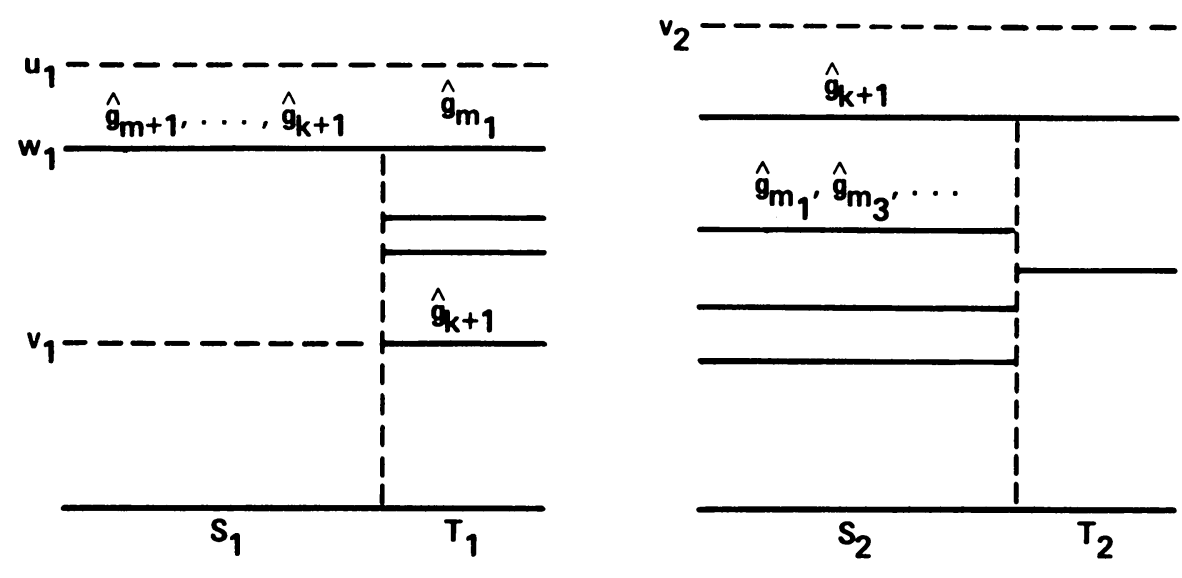

FIGURE IV. Representation of $\left\{\hat{g}_{j}\right\}_{j=m+1}^{k+1} \in G$ over $A_{1}=S_{1} \cup T_{1}$ and $A_{2}=S_{2} \cup T_{2}$ if $1 \in I, 3 \in I$, and $2 \in I^{\prime}$.

$A_{p}$, whereas $g_{j}$ only increases on $S_{p}$ for $j=m+1, \ldots, k$. Thus, some of the $g_{j}$ 's for $j=m+1, \ldots, k$ will eventually increase to $g_{k+1}$ on $S_{p}$ (unless $g_{k+1}$ reaches $v_{i}$ on all $A_{i}$ first for all $i=1, \ldots, m$, in which case Lemma 3.4 finishes the proof with $J=\{k+1\}$ ); this will be done in such a way that when this happens, a group of the $g_{j}$ 's representing the largest on $T_{i}, i \in I$, reach $g_{k+1}$ at the same time, so again Lemma 3.4 can be applied to finish the proof.

A complication which may arise in this "dynamic" approach is the following. The $g_{j}$ 's that were at one time below the group of largest functions on $S_{p}, p \in I^{\prime}$, are repeatedly reaching and then joining the group of functions largest there (not just when $\left.g_{j}\right|_{T_{i}}$ is picked up going down for some $i \in I$, as it might first appear), so the rates must be adjusted to keep a (possibly new) group of representatives together. This could conceivably lead to a possible "Zeno's paradox" situation, where infinitely many readjustments have to be made before reaching the goal. For the formal proof, which now begins, an indirect approach avoids this complication.

Next, let

$$
G=\left\{\left\{\hat{g}_{j}\right\}_{j=m+1}^{k+1} \subseteq \mathcal{G}_{S} \text { which satisfy (3.7) and (3.19)-(3.21) below }\right\} .
$$

(3.19) For each $j=m+1, \ldots, k+1,\left.\hat{g}_{j}\right|_{A_{i}}=\left.g_{j}\right|_{A_{i}}$ for $i=m+1, \ldots, n$; and for each $j=m+1, \ldots, k,\left.\hat{g}_{j}\right|_{T_{i}} \leq\left. g_{j}\right|_{T_{i}}$ for all $i=1, \ldots, m$.

$$
v_{i} \geq\left.\hat{g}_{k+1}\right|_{A_{i}}=\text { constant } \geq\left.\hat{g}_{j}\right|_{A_{i}} \text { for all } i \in I^{\prime} \text { and } j=m+1, \ldots, k \text {. }
$$

(3.21) For each $i \in I,\left.\hat{g}_{k+1}\right|_{T_{i}}=v_{i}$ and there exists $w_{i} \in\left[v_{i}, u_{i}\right]$ and $m_{i} \in\{m+1, \ldots, k\}$ such that $\left.\hat{g}_{m_{i}}\right|_{A_{i}}=$ constant $=w_{i}=\left.\hat{g}_{j}\right|_{S_{i}} \geq$ $\left.\hat{g}_{j}\right|_{T_{i}}$ for $j=m+1, \ldots, k+1$, and if $w_{i}>v_{i}$ and $\lambda\left(T_{i}\right)>0$, then $\left.\hat{g}_{m_{i}}\right|_{S_{p}}=\left.\left(\bigvee_{j=m+1}^{k} \hat{g}_{j}\right)\right|_{S_{p}}$ for all $p \in I^{\prime}$.

See Figure IV. 
(These are systems $\left\{\hat{g}_{j}\right\}_{j=m+1}^{k+1}$ of $k-m$ functions (in $\mathcal{G}_{S}$ ) which coincide on $S_{i}$ for $i \in I$ and satisfy (3.7); for which $\hat{g}_{j}$ is $\leq g_{j}$ on $Q$ for $j=m+1, \ldots, k$; for which $\hat{g}_{j}=g_{j}$ on $A_{i}$ for $i=m+1, \ldots, n$ and $j=m+1, \ldots, k+1$; and for which a set of "top representatives" of the functions on $T_{i}$ (for all $i \in I$ ) are merged on $S_{p}$ and $\geq$ the other $\hat{g}_{j}$ 's on $S_{p}$, for all $p \in I^{\prime}$.)

From (3.18) and the fact that $\left\{g_{j}\right\}_{j=m+1}^{k}$ satisfy the hypothesis of (3.15), it follows that $\left\{g_{j}\right\}_{j=m+1}^{k+1} \in G$ so $G \neq \varnothing$. Observe that if $w_{i}=v_{i}$, or $\lambda\left(T_{i}\right)=0$, then $\hat{g}_{k+1}$ serves as a representative for the top on $T_{i}$; if $\lambda\left(T_{i}\right)=0$, then without loss of generality $T_{i}=\varnothing$.

If there exists $\left\{\hat{g}_{j}\right\}_{j=m+1}^{k+1} \in G$ for which $w_{i}=\left.\hat{g}_{m_{i}}\right|_{A_{i}}=v_{i}$ for all $i \in I$ with $\lambda\left(T_{i}\right)>0\left(\left\{w_{i}\right\}\right.$ and $\left\{m_{i}\right\}$ as in (3.21)), then $\left.\hat{g}_{k+1}\right|_{A_{i}}$ is constant for each $i \in I$. Thus $\left.\hat{g}_{k+1}\right|_{A_{i}}$ is constant for all $i=1, \ldots, m$, and $\hat{g}_{k+1}$ dominates $\hat{g}_{j}, j=m+1, \ldots, k$, on $\bigcup_{i=1}^{m} A_{i}$, so again Lemma 3.4 can be applied, with $J=\{k+1\}$, to complete the proof of (3.15). Consequently, for the remainder of this section, assume

(3.22) $w_{i}>v_{i}$ and $\lambda\left(T_{i}\right)>0$, for at least one $i \in I$, for each $\left\{\hat{g}_{j}\right\}_{j=m+1}^{k+1} \in G$.

Observe that for all indices $i \in I$ for which $w_{i}>v_{i}$ and $\lambda\left(T_{i}\right)>0$, the $\hat{g}_{m_{i}} \mid S_{p}$ values coincide for each $p \in I^{\prime}$, by (3.21). Thus the following definition makes sense; it is a measure of how far the group of "top $T_{i}$ representatives" $\left\{\hat{g}_{m_{i}}\right\}$ is from coinciding with $\hat{g}_{k+1}$ on $S_{p}$ for all $p \in I^{\prime}$, for the best possible $\left\{\hat{g}_{j}\right\}_{j=m+1}^{k+1}$ in $G$.

DEFINITION.

$$
\beta=\inf \left\{\sum_{p \in I^{\prime}} \int_{S_{p}}\left(\hat{g}_{k+1}-\hat{g}_{m_{i}}\right) d \lambda:\left\{\hat{g}_{j}\right\}_{j=m+1}^{k+1} \in G\right\}
$$

where, for a given $\left\{\hat{g}_{j}\right\}_{j=m+1}^{k+1} \in G$, the index $i$ related to $\hat{g}_{m_{i}}$ is any element of $I$ for which $w_{i}>v_{i}$ and $\lambda\left(T_{i}\right)>0$.

LEMMA 3.6. The infimum $\beta$ is attained for some element of $G$.

Proof. Choose $\left\{\hat{g}_{j}^{q}\right\}_{j=m+1}^{k+1} \in G, q=1,2, \ldots$, such that

$$
\sum_{p \in I^{\prime}} \int_{S_{p}}\left(\hat{g}_{k+1}^{q}-\hat{g}_{m(i, q)}^{q}\right) d \lambda=\beta_{q} \searrow \beta \quad \text { as } q \rightarrow \infty,
$$

where $m(i, q), i \in I$, are the $m_{i}$ 's of (3.21) for $\left\{\hat{g}_{j}^{q}\right\}_{j=m+1}^{k+1}$. For each $i \in I$, since $m$ and $k$ are finite and $m+1 \leq m(i, q) \leq k$, it may be assumed by passing to subsequences that $m(i, q)=m_{i}$ is the same for all $q=1,2, \ldots$. By passing to further subsequences, it may be assumed that $\hat{g}_{j}^{q}$ converges uniformly to a limit function $\hat{g}_{j}$, as $q \rightarrow \infty$, for each $j=m+1, \ldots, k+1$ (since the functions are constant on the atoms of a finite algebra). It clearly follows that $\left\{\hat{g}_{j}\right\}_{j=m+1}^{k+1} \in G$ and that $\beta$ is attained for this element of $G$, which completes the proof of the lemma.

LEMMA 3.7. $\beta=0$.

PROOF. Let $\left\{\hat{g}_{j}\right\}_{j=m+1}^{k+1}$ be the element of $G$ attaining the infimum $\beta$ (the existence of which is guaranteed by Lemma 3.6), and suppose by way of contradiction that $\beta>0$. Let

$$
I_{0}=\left\{i \in I: w_{i}>v_{i} \text { and } \lambda\left(T_{i}\right)>0\right\}
$$



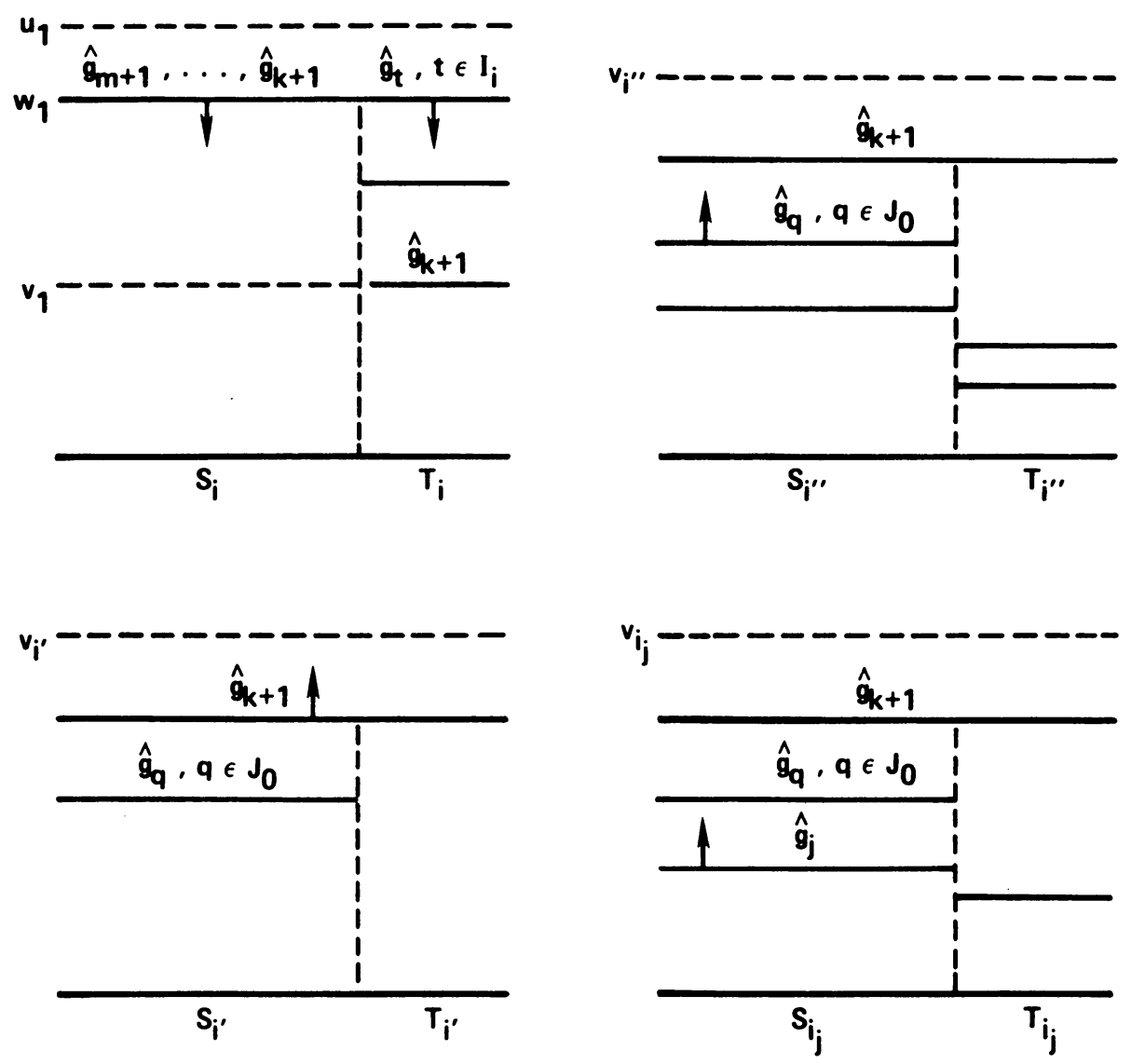

FIGURE V. Representation of modification to $\left\{\hat{g}_{j}\right\}_{j=m+1}^{k+1}$ in the proof of Lemma 3.7, where $i \in I_{0}, i^{\prime} \in I^{\prime}, i^{\prime \prime} \in I^{\prime}$, and $j \in\{m+1, \ldots, k+1\} \backslash J_{0}$ with associated $i_{j} \in I^{\prime}$ (from (3.26)). Observe that $v_{i^{\prime \prime}}$ could equal $\left.\hat{g}_{k+1}\right|_{S_{i^{\prime \prime}}}$ and $\left.\hat{g}_{q}\right|_{S_{i^{\prime}}}$ could equal $\left.\hat{g}_{k+1}\right|_{S_{i^{\prime}}}$ for $q \in J_{0}$.

Then

(3.23) $I_{0} \neq \varnothing$;

(3.24) $\left.\hat{g}_{k+1}\right|_{A_{i^{\prime}}}<v_{i^{\prime}}$ for some $i^{\prime} \in I^{\prime}$; and

(3.25) for some $i^{\prime \prime} \in I^{\prime},\left.\left(\bigvee_{j=m+1}^{k} \hat{g}_{j}\right)\right|_{S_{i^{\prime \prime}}}<\left.\hat{g}_{k+1}\right|_{S_{i^{\prime \prime}}}$.

Statement (3.23) follows from (3.22); for (3.24) recall (3.16), (3.20), and (3.21), and use (3.23), normalization, and the fact that $\hat{g}_{k+1}=f_{k+1}$ on $\bigcup_{i=m+1}^{n} A_{i}$; and (3.25) follows from the assumption $\beta>0$. For $i \in I_{0}$, let

$$
I_{i}=\left\{j \in\{m+1, \ldots, k\}:\left.\hat{g}_{j}\right|_{T_{i}}=\left.\left(\bigvee_{q=m+1}^{k} \hat{g}_{q}\right)\right|_{T_{i}}\right\}
$$


that is, $I_{i}$ is the set of $j$ for which $\hat{g}_{j}$ is a top representative on $T_{i}$. Also, let

$$
J_{0}=\left\{j \in\{m+1, \ldots, k\}:\left.\hat{g}_{j}\right|_{S_{i}}=\left.\left(\bigvee_{q=m+1}^{k} \hat{g}_{q}\right)\right|_{S_{i}} \text { for every } i \in I^{\prime}\right\} ;
$$

that is, $J_{0}$ is the set of indices $j$ for which $\hat{g}_{j}$ is part of the top group of the $\hat{g}_{q}$ 's on $S_{i}(m+1 \leq q \leq k)$ for every $i \in I^{\prime}$. Observe that by the definition of $G, m_{i} \in I_{i} \cap J_{0}$ for every $i \in I_{0}$ (so $I_{i} \cap J_{0} \neq \varnothing$ for all $i \in I_{0}$ ). In addition,

(3.26) for each $j \in\{m+1, \ldots, k\} \backslash J_{0}$ there exists $i_{j} \in I^{\prime}$ for which $\left.\hat{g}_{j}\right|_{S_{i_{j}}}<$

$$
\left.\left(\bigvee_{q=m+1}^{k} \hat{g}_{q}\right)\right|_{S_{i_{j}}}
$$

New functions (which will again be denoted $\left\{\hat{g}_{j}\right\}_{j=m+1}^{k+1}$ ) will be produced by decreasing the $\left.\hat{g}_{j}\right|_{S_{i}}, j=m+1, \ldots, k+1$, and $\hat{g}_{j} \mid T_{i}, j \in I_{i}$, by small amounts $\Delta w_{i}$ for all $i \in I_{0}$; and, to maintain normalization, by increasing $\hat{g}_{j} \mid s_{i^{\prime \prime}}$ for all $j \in J_{0}$, and $\left.\hat{g}_{k+1}\right|_{A_{i^{\prime}}}$, and also $\left.\hat{g}_{j}\right|_{s_{i_{j}}}$ for each $j \in\{m+1, \ldots, k\} \backslash J_{0}$ and associated index $i_{j} \in I^{\prime}$ from (3.26) (see Figure V). By choosing $\varepsilon>0$ sufficiently small, and requiring that the "decreasing" increments $\Delta w_{i}$ satisfy $0 \leq \Delta w_{i} \leq \varepsilon$ for all $i \in I_{0}$, after the modification the new $\hat{g}_{j}$ 's continue to satisfy

(i) $\left.\hat{g}_{j}\right|_{T_{i}}>\max \left\{\left.\hat{g}_{u}\right|_{T_{i}}: m+1 \leq u \leq k+1, u \notin I_{i}\right\}$ for all $j \in I_{i}, i \in I_{0}$

(ii) $\left.\hat{g}_{j}\right|_{S_{i}} \leq\left.\left(\bigvee_{j \in J_{0}} \hat{g}_{j}\right)\right|_{S_{i}}$ for all $i \in I^{\prime}$ and all $j \in\{m+1, \ldots, k+1\} \backslash J_{0}$;

(iii) $\left.\left(\bigvee_{j=m+1}^{k} \hat{g}_{j}\right)\right|_{S_{i^{\prime \prime}}}<\left.\hat{g}_{k+1}\right|_{S_{i^{\prime \prime}}}$;

(iv) $\left.\hat{g}_{k+1}\right|_{A_{i^{\prime}}} \leq v_{i^{\prime}}$.

In (3.27), (i) follows since $i \in I_{0}$, from (3.22) and from the fact that $\hat{g}_{m_{i}} \mid T_{i}>$ $\max \left\{\left.\hat{g}_{u}\right|_{T_{i}}: m+1 \leq u \leq k+1, u \notin I_{i}\right\}$ for $i \in I_{0}$; (ii) follows since by (3.26) there exists a $\delta>0$ such that for every $j \in\{m+1, \ldots, k\} \backslash J_{0},\left.\hat{g}_{j}\right|_{S_{i_{j}}}+\delta \leq\left.\left(\bigvee_{q=m+1}^{k} \hat{g}_{q}\right)\right|_{s_{i_{j}}}$, so taking $\varepsilon<\delta(\lambda(S))^{-1} \min \left\{\lambda\left(S_{p}\right): p \in I^{\prime}\right\}$ will work; (iii) follows from (3.25) and the fact that $\lambda\left(S_{i^{\prime \prime}}\right)>0$; and (iv) follows from (3.24) and the fact that $\lambda\left(A_{i^{\prime}}\right)>0$ (see Figure V).

It is next claimed that

(3.28) there exist indices $n_{i} \in I \cap J_{0}$, and $0 \leq \Delta w_{i} \leq \varepsilon$, for each $i \in I_{0}$, not all $\Delta w_{i}$ being zero, such that if the modifications described between (3.26) and (3.27) are completed, then $\hat{g}_{n_{i}} \mid s_{i^{\prime \prime}}$ increases the same amount for each $i \in I_{0}$, and this amount is greater than or equal to the amount that $\left.\hat{g}_{j}\right|_{s_{i^{\prime \prime}}}$ increases for other $j \in J_{0}$ (thus $\left.\hat{g}_{n_{i}}\right|_{S_{i^{\prime \prime}}}, i \in I_{0}$, stay together after the change and on top among the $\left.\left.\hat{g}_{j}\right|_{S_{i^{\prime \prime}}}, j \in J_{0}\right)$. 
Assume (3.28) for the moment, and complete the proof of the lemma as follows. After the modifications are completed, the new system $\left\{\hat{g}_{j}\right\}_{j=m+1}^{k+1}$ is in $G$ also, with indices $\left\{n_{i}, i \in I_{0}\right\}$ taking the role of $\left\{m_{i}, i \in I_{0}\right\}$ in (3.21) (from choice of $\varepsilon>0$, and $0 \leq \Delta w_{i} \leq \varepsilon$, so that (3.27) and (3.28) hold). Next, since $\Delta w_{i_{0}}>0$ and $\lambda\left(T_{i_{0}}\right)>0$ for some $i_{0} \in I_{0}$, it is seen that $\int_{A_{i_{0}}} \hat{g}_{n_{i_{0}}} d \lambda$ has decreased strictly more than $\int_{S_{i_{0}}} \hat{g}_{k+1} d \lambda$ has decreased in changing to the new functions (namely by amount $\left.\Delta w_{i_{0}} \lambda\left(T_{i_{0}}\right)\right)$; and $\int_{A_{i}} \hat{g}_{n_{i_{0}}} d \lambda$ decreases at least as much as $\int_{S_{i}} \hat{g}_{k+1} d \lambda$ decreases for every other $i \in I_{0}$. Thus, by normalization, $\sum_{p \in I^{\prime}} \int_{S_{p}}\left(\hat{g}_{k+1}-\hat{g}_{n_{i_{0}}}\right) d \lambda$ is strictly less for the new functions than for the old functions, which was equal to $\beta$; this contradicts the definition of $\beta$ as infimum, and completes the proof, assuming (3.28).

It remains only to prove $(3.28)$, which will be done by induction. Enumerate $I_{0}=\left\{i_{1}, \ldots, i_{s}\right\}$, and let $1 \leq r<s$. The induction hypothesis is:

(3.29) for each $i=i_{1}, \ldots, i_{r}$ there exist $n_{i} \in I_{i} \cap J_{0}$ and $0 \leq d_{i} \leq \varepsilon$, not all $d_{i}$ being zero, such that if $\left.\hat{g}_{j}\right|_{S_{j}}, j=m+1, \ldots, k+1$, and $\left.\hat{g}_{j}\right|_{T_{i}}, j \in I_{i}$, are decreased by $d_{i}$, for $i=i_{1}, \ldots, i_{r}$, and (to preserve normalization) $\left.\hat{g}_{j}\right|_{S_{i^{\prime \prime}}}$ for $j \in J_{0}$, and $\left.\hat{g}_{k+1}\right|_{A_{i^{\prime}}}$, and $\left.\hat{g}_{j}\right|_{S_{i_{j}}}$ for each $j \in\{m+1, \ldots, k\} \backslash J_{0}$ and associated $i_{j} \in I^{\prime}$, are all increased, then $\left.\hat{g}_{n_{i}}\right|_{S_{i^{\prime \prime}}}$ increases by the same amount, say $\Delta$, for each $i=i_{1}, \ldots, i_{r}$, and furthermore,

this is greater than or equal to the amount that $\left.\hat{g}_{j}\right|_{S_{i^{\prime \prime}}}$ increases for other $j \in J_{0}$.

It is clear that (3.29) holds for $r=1$ (by letting $n_{i_{1}}$ be any element of $I_{i_{1}} \cap J_{0}$, and $d_{i_{1}}=\varepsilon$ ). Assume (3.29) holds for $1 \leq r \leq s$.

Consider $I_{i_{r+1}} \cap J_{0}$. If there exists $q \in I_{i_{r+1}} \cap J_{0}$ such that decreasing $\left.\hat{g}_{q}\right|_{S_{i}}$ (and also $\hat{g}_{q} \mid T_{i}$ if $q \in I_{i}$ ) by $d_{i}$ for each $i=i_{1}, \ldots, i_{r}$ causes $\left.\hat{g}_{q}\right|_{S_{i^{\prime \prime}}}$ to increase by exactly $\Delta$ (it could not be more by $(*)$ ), then let $n_{i_{r+1}}=q$ and $d_{i_{r+1}}=0$, and (3.29) holds with $r+1$ in place of $r$. If no such $q$ exists in $I_{i_{r+1}} \cap J_{0}$, choose $q \in I_{i_{r+1}} \cap J_{0}$ such that the increase in $\left.\hat{g}_{q}\right|_{S_{i^{\prime \prime}}}$ caused by decreasing $\left.\hat{g}_{q}\right|_{S_{i}}$ (and $\left.\hat{g}_{q}\right|_{T_{i}}$ if $q \in I_{i}$ ) by amount $d_{i}$ for $i=i_{1}, \ldots, i_{r}$ is as large as possible, say $\Delta^{\prime}$ (which is $<\Delta$ ).

Let $n_{i_{r+1}}=q$. Suppose $\left.\hat{g}_{j}\right|_{S_{i_{r+1}}}, j=m+1, \ldots, k+1$, and $\left.\hat{g}_{j}\right|_{T_{i_{r+1}}}, j \in I_{i_{r+1}}$, are decreased by $\varepsilon$, and (to preserve normalization) $\hat{g}_{j}$ are increased as before. Then, since $n_{i} \notin I_{i_{r+1}}$ for $i=i_{1}, \ldots, i_{r}$ (otherwise the proof is complete by the previous paragraph), $\hat{g}_{n_{i}}$ have been decreased only on $S_{i_{r+1}}$ and not on $T_{i_{r+1}}$ for each $i=i_{1}, \ldots, i_{r}$; so the increase caused (by preserving normalization) in $\left.\hat{g}_{n_{i}}\right|_{S_{i^{\prime \prime}}}$, say $\alpha$, would be the same for each $i=i_{1}, \ldots, i_{r}$, and strictly less than the increase in $\left.\hat{g}_{n_{i_{r+1}}}\right|_{S_{i^{\prime \prime}}}$, say $\alpha^{\prime}$ (since $n_{i_{r+1}} \in I_{i_{r+1}}$ and thus $\hat{g}_{n_{i_{r+1}}}$ is decreased on $T_{i_{r+1}}$ as well, which has positive measure by definition of $I_{0}$ ).

It follows that these modifications may be averaged, keeping all the $\hat{g}_{n_{i}}, i=$ $i_{1}, \ldots, i_{r}, i_{r+1}$, together on $S_{i^{\prime \prime}}$ ! More precisely, there exists $\mu$ with $0<\mu<1$ such that $\mu \Delta+(1-\mu) \alpha=\mu \Delta^{\prime}+(1-\mu) \alpha^{\prime}$ (which follows simply from $\alpha^{\prime}>\alpha$ and $\left.\Delta^{\prime}<\Delta\right)$. Let

$$
\begin{aligned}
\Delta w_{i} & =\mu d_{i} \quad \text { for } i=i_{1}, \ldots, i_{r} \\
& =(1-\mu) \varepsilon \quad \text { for } i=i_{r+1}
\end{aligned}
$$


Then the decrease in $\left.\hat{g}_{j}\right|_{S_{i}}, j=m+1, \ldots, k+1$, and $\left.\hat{g}_{j}\right|_{T_{i}}, j \in I_{i}$, by the amount $\Delta w_{i}$ of $(3.30)$ for $i=i_{1}, \ldots, i_{r+1}$, causes an increase in $\left.\hat{g}_{n_{i}}\right|_{S_{i^{\prime \prime}}}$, for each $i=i_{1}, \ldots, i_{r}$, of $\mu \Delta+(1-\mu) \alpha$, and an increase in $\left.\hat{g}_{n_{i_{r+1}}}\right|_{S_{i^{\prime \prime}}}$ of $\mu \Delta^{\prime}+(1-\mu) \alpha^{\prime}$, the same amount. Furthermore, if $j \in J_{0} \backslash I_{i_{r+1}}$, then $\hat{g}_{j}$ is not decreased on $T_{i_{r+1}}$, so the decrease of $\hat{g}_{j}$ by the amount $(1-\mu) \varepsilon$ on $S_{i_{r+1}}$ causes an increase in $\left.\hat{g}_{j}\right|_{S_{j^{\prime \prime}}}$ which is the same as that for each $\hat{g}_{n_{i}}, i=i_{1}, \ldots, i_{r}$, namely $(1-\mu) \alpha$. It follows that for these $j \in J_{0} \backslash I_{i_{r+1}}$, all these decreases cause an increase in $\left.\hat{g}_{j}\right|_{S_{i^{\prime \prime}}}$ which is $\leq \mu \Delta+(1-\mu) \alpha$ (recall (*) in (3.29)). On the other hand, if $j \in J_{0} \cap I_{i_{r+1}}$, then by the choice of $n_{i_{r+1}}$, the decreases of $\hat{g}_{j}$ by $\mu d_{i}$ on $S_{i}$ (and also on $T_{i}$ if $j \in I_{i}$ ) for $i=i_{1}, \ldots, i_{r}$ cause an increase in $\left.\hat{g}_{j}\right|_{S_{i^{\prime \prime}}}$ which is less than or equal to $\mu \Delta^{\prime}$; and the decrease of $\hat{g}_{j}$ by the amount $(1-\mu) \varepsilon$ on both $S_{i_{r+1}}$ and $T_{i_{r+1}}$ causes an increase in $\left.\hat{g}_{j}\right|_{S_{i^{\prime \prime}}}$ which is the same as that for $\hat{g}_{n_{i_{r+1}}}$, namely $(1-\mu) \alpha^{\prime}$. So it follows that for these $j \in J_{0} \cap I_{i_{r+1}}$, all these decreases cause an increase in $\left.\hat{g}_{j}\right|_{S_{i^{\prime \prime}}}$ which is $\leq \mu \Delta^{\prime}+(1-\mu) \alpha^{\prime}$.

Thus induction hypothesis (3.29) holds with $r+1$ in place of $r, n_{i_{r+1}}=q$, and $\Delta w_{i}$ (of (3.10)) in the role of $d_{i}, i=i_{1}, \ldots, i_{r+1}$. This completes the proof of (3.28), and the proof of Lemma 3.7.

PROOF OF PROPOSITION 3.5. By the induction step, it is enough to establish (3.15), which is seen to follow from Lemmas 3.6 and 3.7 as follows. Let $\left\{\hat{g}_{j}\right\}_{j=m+1}^{k+1} \in$ $G$ attain the infimum $\beta=0$. Use $\hat{g}_{k+1}$ as the "top representative" on $A_{i}$ for $i \in I^{\prime}$ and also for those $i \in I$ for which $w_{i}=v_{i}$ or $\lambda\left(T_{i}\right)=0$; and use $\hat{g}_{m_{i}}$ as the "top representative" for the other $i \in I$. By the definition of $G$ and the fact that $\beta=0$, all these "representatives" coincide on $S_{i}$, for all $i=1, \ldots, m$, and again Lemma 3.4 can be applied to yield (3.15). (More precisely, use Lemma 3.4 with $J=\{m+1, \ldots, k+1\} ; h_{j}=\hat{g}_{j}$ for $j=m+1, \ldots, k+1$, and $=f_{j}$ for $j=k+2, \ldots, n ; j_{i}=k+1$ for $i \in I^{\prime}$ and also for those $i \in I$ for which $w_{i}=v_{i}$ or $\lambda\left(T_{i}\right)=0$, and $j_{i}=m_{i}$ for the other $i \in I$; and $u_{i}$ (of Lemma 3.4) $=\left.\hat{g}_{k+1}\right|_{A_{i}}$ for $i \in I^{\prime}$ and for those $i \in I$ for which $w_{i}=v_{i}$ or $\lambda\left(T_{i}\right)=0$, and $=w_{i}$ for the other $i \in I$. Observe that the $h_{j}$ 's satisfy $\left.h_{j}\right|_{Q} \leq\left. f_{j}\right|_{Q}$ for $j=m+1, \ldots, k+1$, and hence the functions $\left\{\tilde{g}_{j}\right\}_{j=m+1}^{k+1}$ guaranteed by Lemma 3.4 satisfy (3.13), and thus also satisfy (3.14).)

4. Proof of main results and the extremal distributions. The upper bound in the conclusion of Theorem 1.1 follows since

$$
\min _{1 \leq i \leq n} \mu_{i}\left(S_{i}\right) \leq n^{-1} \sum_{i=1}^{n} \mu_{i}\left(S_{i}\right) \leq n^{-1}\left(\bigvee_{i=1}^{n} \mu_{i}\right)(S)=n^{-1} M
$$

for all partitions $\left\{S_{i}\right\}_{i=1}^{n}$ of $S$. The lower bound is easily seen to follow from Theorem 1.2 by taking $\lambda=\sum_{i=1}^{n} \mu_{i}$, which is clearly nonatomic, and $f_{i}=d \mu_{i} / d \lambda$ for $i=1, \ldots, n$.

ProOF OF THEOREM 1.2. The proof is by induction on $n$. For $n=1$, the conclusion is trivial, since $M=1$ and the only possible partition is $S_{1}=S$. Assume the induction hypothesis (3.1), and let $\left\{f_{i}\right\}_{i=1}^{n} \in \mathcal{F}_{S}^{n}$ satisfy (3.2)-(3.4), where $1 \leq m<n$ (if $m=n$, the theorem is proved).

The $\left\{g_{j}\right\}_{j=m+1}^{n}$ guaranteed by Proposition 3.5 satisfy $(3.14)$ for $j=m+1, \ldots, n$ and the hypotheses of Proposition 3.3. Then the partition $\left\{S_{j}\right\}_{j=m+1}^{n}$ of $Q$ guaranteed by Proposition 3.3 completes the proof, since $\int_{S_{j}} f_{j} d \lambda \geq \int_{S_{j}} g_{j} d \lambda \geq$ $(n+1-M)^{-1}$ for all $j=m+1, \ldots, n$. 


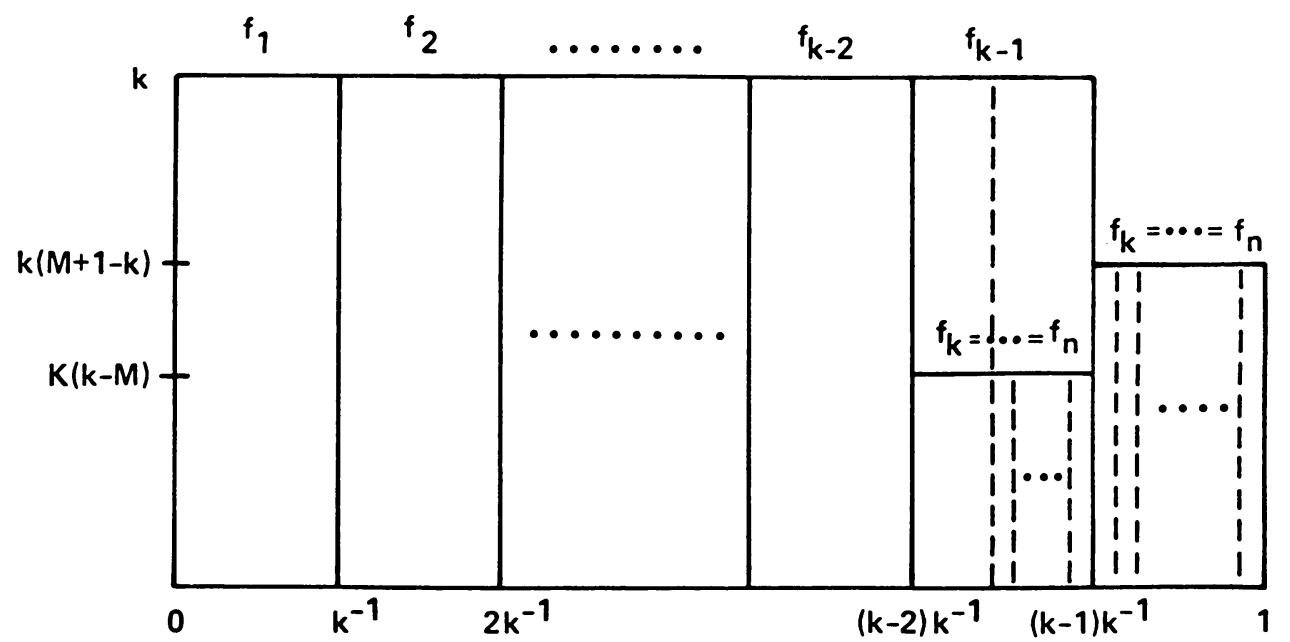

FIGURE VI. Representation of $\left\{f_{j}\right\}_{j=1}^{n}$ for the extremal case of $1 \leq M<n$ in Example 4.1.

The next two examples show that the inequalities in the conclusion of Theorem 1.1 can both be attained for all $n$ and $M$. Recall that $1 \leq M \leq n$.

EXAMPLE 4.1. (Extremal distributions for the lower bound.)

Case $1 . M=n$. Any $\left\{\mu_{i}\right\}_{i=1}^{n}$ with disjoint supports satisfy $v^{*}\left(\mu_{1}, \ldots, \mu_{n}\right)=1$.

Case 2. $M<n$ (see Figure VI). Let $[M]=$ largest integer $\leq M$ and let $k=[M]+1$. For $j=1, \ldots, k-1$, let $f_{j}(x)=k$ for $x \in\left[(j-1) k^{-1}, j k^{-1}\right)$, and $=0$ otherwise; and for $j=k, \ldots, n$, let $f_{j}(x)=k(k-M)$ for $x \in\left[(k-2) k^{-1},(k-1) k^{-1}\right)$, $=k(M+1-k)$ for $x \in\left[(k-1) k^{-1}, 1\right)$, and $=0$ otherwise. Take $(S, B)=$ $([0,1), B([0,1)))$, and $d \mu_{i}=f_{i} d \lambda$ for each $i=1, \ldots, n$, where $\lambda$ is Lebesgue measure.

Let

$$
\begin{aligned}
S_{j}= & {\left[(j-1) k^{-1}, j k^{-1}\right) \quad \text { for } j=1, \ldots, k-2 ; } \\
S_{k-1}= & {\left[(k-2) k^{-1}, k-2+(n+1-M)^{-1} k^{-1}\right) ; } \\
S_{k-r}= & {\left[(k-2) k^{-1}+\left(1+r(n-M)(n-k+1)^{-1}\right)(n+1-M)^{-1} k^{-1},\right.} \\
& \left.(k-2)^{k-1}+\left(1+(r+1)(n-M)(n-k+1)^{-1}\right)(n+1-M)^{-1} k^{-1}\right) \\
& \cup\left[(k-1) k^{-1}+r(n-k+1)^{-1} k^{-1},\right. \\
& \left.(k-1) k^{-1}+(r+1)(n-k+1)^{-1} k^{-1}\right)
\end{aligned}
$$

for $r=0, \ldots, n-k$. Then $\int_{S_{j}} f_{j} d \lambda=1$ for $j=1, \ldots, k-2$, and $=(n+1-M)^{-1}$ for $j=k-1, k, \ldots, n$; so $v^{*}\left(\mu_{1}, \ldots, \mu_{n}\right)=v^{*}\left(f_{1}, \ldots, f_{n} ; \lambda\right)=(n+1-M)^{-1}$, since it is clear that no other partition can do better.

EXAMPLE 4.2. (Extremal distributions for the upper bound.) The case $n=1$ is trivial, so suppose $n>1$, and let $p=(n-M)(n-1)^{-1}$. For $j=1, \ldots, n$, let $f_{j}(x)=n$ for $x \in\left[(j-1)(1-p) n^{-1}, j(1-p) n^{-1}\right),=1$ for $x \in[1-p, 1)$, and $=0$ otherwise. For $j=1, \ldots, n$, let

$$
S_{j}=\left[(j-1)(1-p) n^{-1}, j(1-p) n^{-1}\right) \cup\left[1-p+(j-1) p n^{-1}, 1-p+j p n^{-1}\right) .
$$

Then

$$
v^{*}\left(f_{1}, \ldots, f_{n} ; \lambda\right)=v^{*}\left(\mu_{1}, \ldots, \mu_{n}\right)=M n^{-1} \text { for } j=1, \ldots, n
$$

(where again $d \mu_{i}=f_{i} d \lambda$ ). 
It is clear that the functions $v^{*}$ and $M$ are continuous functions of the measures $\mu_{1}, \ldots, \mu_{n}$ (strong topology, total-variation norm); the next example shows that $v^{*}$ is in general neither convex nor concave.

EXAMPLE 4.3. Take $\mu_{1}=$ Lebesgue measure on $[0,1), \mu_{2}=$ Lebesgue measure on $[1,2)$, and $\mu_{3}=2^{-1}$ (Lebesgue measure on $[0,2)$ ). Then it is easy to check that

$$
v^{*}\left(\mu_{1}, \frac{1}{2} \mu_{1}+\frac{1}{2} \mu_{2}\right)=\frac{2}{3}<\frac{3}{4}=\frac{1}{2} v^{*}\left(\mu_{1}, \mu_{1}\right)+\frac{1}{2} v^{*}\left(\mu_{1}, \mu_{2}\right),
$$

and

$$
v^{*}\left(\frac{1}{2}\left(\mu_{1}+\mu_{3}\right), \mu_{3}\right)=\frac{4}{5}>\frac{7}{12}=\frac{1}{2} v^{*}\left(\mu_{1}, \mu_{3}\right)+\frac{1}{2} v^{*}\left(\mu_{3}, \mu_{3}\right) .
$$

The final example in this section calculates $v^{*}$ and the upper and lower bounds given by Theorem 1.1 for two explicit common measures, a uniform and a triangular distribution.

EXAMPLE 4.4. Suppose $\mu_{1}$ is Lebesgue measure on $[0,1]$, and $d \mu_{2}$ is $f_{2} d \lambda$ where $f_{2}(x)=2 x$. Then

$$
\frac{1}{(n-M+1)}=\frac{4}{7}<v^{*}=\frac{\sqrt{5}-1}{2}<\frac{5}{8}=M n^{-1} .
$$

5. Applications to discrete allocation problems. A "fair-division" question investigated by Demko and Hill [2] is the following. Suppose $k$ indivisible objects must be distributed among $n$ people whose values of the objects may differ. In general there is not a deterministic assignment of objects to people so that each person receives what he considers $1 / n$ of the total value (for example if $k<n$, or if $k \geq n$ but everyone agrees that the same $k-n-1$ objects are nearly worthless), but there is always a randomized or lotterized solution. That is, there is always a way of assigning objects to people randomly, so that the expected return to each person is at least $1 / n$ according to his own values. Two such solutions are: roll an $n$-die once and if $j$ comes up, then person $j$ gets everything; or, for each object roll an $n$-die once, etc. Both of these solutions give expected return of exactly $1 / n$ to each person, but if the (proportionate) values assigned by the $n$ people to the $k$ objects are not all identical, then an even better solution is possible in which each participant has an expected return of strictly more than $1 / n$. In fact, at least $(n-M+1)^{-1}$ can always be attained, where $M$ is again the "cooperative" or "total" value. The mathematical formulation is as follows.

Let $A=\left(a_{i j}\right)$ be any $n \times k$ stochastic matrix (i.e., $a_{i j} \geq 0$ for all $i=1, \ldots, n, j=$ $1, \ldots, k$, and $\sum_{j=1}^{k} a_{i j}=1$ for all $\left.i=1, \ldots, n\right)$ and let $S_{k, n}$ denote the set of $k \times n$ stochastic matrices, with element $p=\left(p_{j i}\right) \in S_{k, n}$. Analogous to (1.1)-(1.2), define

$$
\begin{gathered}
M(A)=\sum_{j=1}^{k} \max _{1 \leq i \leq n} a_{i j}, \\
v^{*}(A)=\sup \left\{\min _{1 \leq i \leq n} \sum_{j=1}^{k} a_{i j} p_{j i}:\left(p_{j i}\right) \in S_{k, n}\right\} .
\end{gathered}
$$

The matrix $A=\left(a_{i j}\right)$ represents the "value" matrix; entry $a_{i j}$ is the value of the $j$ th object to person $i$. The matrices $\left(p_{j i}\right)$ represent the "lotteries"; entry $p_{j i}$ is the probability that object $j$ goes to person $i$ in lottery $p$. Again, $M$ and $v^{*}$ represent 
the "cooperative" and "noncooperative" values, respectively, of the $k$ objects to the $n$ people.

THEOREM 5.1. For every $n \times k$ stochastic matrix $A$,

$$
(n+1-M(A))^{-1} \leq v^{*}(A) \leq n^{-1} M(A) .
$$

SKETCH OF PROOF. The upper-bound conclusion is again easy. For the lowerbound conclusion, fix an $n \times k$ stochastic matrix $A=\left(a_{i j}\right)$, and define $f_{1}, \ldots, f_{n} \in$ $L_{1}[0,1)$ by $f_{i}(x)=k a_{i j}$ for $x \in\left[(j-1) k^{-1}, j k^{-1}\right), j=1, \ldots, k$, and $i=1, \ldots, n$. Next, identify lotteries $p$ with partitions $\left\{S_{i}\right\}_{i=1}^{n}$ of $[0,1)$ by $S_{i}=\bigcup_{j=1}^{k} E_{i j}(p)$, where $E_{i j}(p)$ are disjoint subsets of $\left[(j-1) k^{-1}, j k^{-1}\right)$ with Lebesgue measure $k^{-1} p_{j i}$. The lower-bound conclusion is then a straightforward application of Theorem 1.2 , since $M\left(f_{1}, \ldots, f_{m} ; \lambda\right)=M(A)$ and $v^{*}\left(f_{1}, \ldots, f_{n} ; \lambda\right)=v^{*}(A)$.

The next two examples are matrix analogs of Examples 4.1 and 4.2.

EXAMPLE 5.2. (Sharpness of lower bound.) The case $M=n$ is trivial, since no object is valued strictly positively by more than one person; hence $M(A)=n$ and $v^{*}(A)=1$. Suppose, then, that $M<n$, and let $k=[M]+1$, where $[M]=$ the largest integer $\leq M$. Let $A=\left(a_{i j}\right)$ be the $n \times k$ stochastic matrix given by

$$
\begin{aligned}
& a_{i j}=1 \text { for } i=j, \quad=0 \text { for } i \neq j, \text { for } j=1, \ldots, k-2 \\
& a_{i, k-1}=0 \text { for } i=1, \ldots, k-2, \quad=1 \text { for } i=k-1, \quad=k-M \text { otherwise; } \\
& a_{i, k}=0 \text { for } i=1, \ldots, k-1, \quad=M+1-k \text { otherwise. }
\end{aligned}
$$

By constructing $f_{1}, \ldots, f_{n}$ as in the proof of Theorem 5.1 and observing that these $f_{i}$ 's are the functions of Example 4.1, it is immediate that

$$
M(A)=M\left(f_{1}, \ldots, f_{n} ; \lambda\right)=M
$$

and $v^{*}(A)=v^{*}\left(f_{1}, \ldots, f_{n} ; \lambda\right)=(n+1-M)^{-1}$, with maximizing $p$ for $v^{*}(A)$ given by

$$
\begin{aligned}
& p_{j i}=1 \text { for } i=j, \quad=0 \text { for } i \neq j, \text { for } j=1, \ldots, k-2 ; \\
& \begin{array}{r}
p_{k-1, i}=0 \text { for } i=1, \ldots, k-2, \quad=(n+1-M)^{-1} \text { for } i=k-1, \\
=(n-M)(n-k+1)^{-1}(n+1-M)^{-1} \text { otherwise; }
\end{array} \\
& p_{k, i}=0 \text { for } i=1, \ldots, k-1, \quad=(n-k+1)^{-1} \text { otherwise. }
\end{aligned}
$$

Alternatively, a direct argument can be given as follows. By symmetry, there is an optimal lottery in which the last $n-k+1$ people (rows) each get object (column) $k$ with probability $(n-k+1)^{-1}$ and object $k-1$ with some probability $q \leq(n-k+1)^{-1}$. Also, it is clear that person $i$ gets object $i$ with probability one, for $i=1, \ldots, k-2$, so

$$
v^{*}=(M-k+1)(n-k+1)^{-1}+q(k-M)=1-(n-k+1) q
$$

where (for $i=1, \ldots, n-[M]$ ) the first summand in the middle term of $(5.3)$ is the probability person $i$ gets piece $k$ times the value of piece $k$ to person $i$; the second is the probability person $i$ gets object $k-1$ times the value of object $k-1$ to person $i$; and the right term is the probability person $k-1$ gets piece $k-1$ times his value of piece $k-1$. Solving (5.3) for $q$ yields $q=(n-k+1)^{-1}(n-M)(n-M+1)^{-1}$ and $v^{*}=(n-M+1)^{-1}$. 
As the previous example showed, the lower bound in Theorem 5.1 is sharp for all choices of $n, k$, and $M$; such is not the case for the upper bound. For example, if $n=3$ and $k=M=2$, it is easy to see that the upper bound is not attained. If, however, there are more objects than people (i.e., $k>n$ ), then for given $M$ the upper bound is also sharp.

EXAMPLE 5.3. (Sharpness of upper bound.) For $n=1$, the conclusion is trivial, so suppose $n>1$. If $k>n$, let $A=\left(a_{i j}\right)$ be the $n \times k$ stochastic matrix given by

$$
\begin{aligned}
a_{i j} & =(M-1)(n-1)^{-1} \quad \text { for } 1 \leq i=j \leq n \\
& =(n-M)(n-1)^{-1} \quad \text { for } i=1, \ldots, n, \text { and } j=n+1 ; \\
& =0 \text { otherwise. }
\end{aligned}
$$

Then it is easy to check that $M=M(A)$, and that $v^{*}(A)=M n^{-1}$.

6. Applications to a dual problem and to statistical decision theory. In this section a "dual" to the above partitioning problem is identified, and then Theorem 1.1 is applied in this new setting and to a problem in statistical decision theory.

The next proposition, which identifies the dual problem, may be regarded as a restatement of Proposition 2.2 of Dor [3]; its application in another equidistribution problem is found in Hill [7]. The proof given here based on the minimax theorem is due to Shmuel Friedland.

Let $\Delta_{n}$ denote the set of probabilities on $n$ points, that is, $\Delta_{n}=\left\{\left(p_{i}\right)_{i=1}^{n}: p_{i} \geq 0\right.$ and $\left.\sum_{i=1}^{n} p_{i}=1\right\}$, and let $v^{*}$ be as in (1.2). For measures $\mu_{1}, \ldots, \mu_{n}$ on the same measure space, let $\bigvee_{i=1}^{n} \mu_{i}$ denote the maximum of the measures $\mu_{1}, \ldots, \mu_{n}$ (e.g., Dunford and Schwartz [5, p. 162]). (Recall that $\bigvee_{i=1}^{n} \mu_{i}$ may be characterized as the smallest measure which majorizes each of the $\left\{\mu_{i}\right\}$ or defined explicitly by

$$
\left(\bigvee_{i=1}^{n} \mu_{i}\right)(E)=\sup \left\{\sum_{i=1}^{n} \mu_{i}\left(S_{i}\right):\left\{S_{i}\right\}_{i=1}^{n} \in \Pi_{E}\right\} .
$$

Alternatively, $\left(\bigvee_{i=1}^{n} \mu_{i}\right)(E)=\int_{E} \bigvee_{i=1}^{n} f_{i} d \lambda$, where $\lambda=\sum_{i=1}^{n} \mu_{i}$ and $f_{i}=d \mu_{i} / d \lambda$.) Let $\|\mu\|$ denote the total variation $\mu(S)$ of the measure $\mu$. then

PROPOSITION 6.1. If $\mu_{1}, \ldots, \mu_{n}$ are nonatomic probability measures on $(S, B)$,

$$
v^{*}=\min \left\{\left\|\bigvee_{i=1}^{n} p_{i} \mu_{i}\right\|:\left(p_{i}\right)_{i=1}^{n} \in \Delta_{n}\right\} .
$$

PROOF. For convenience this proof will also be given in the $L_{1}$ setting; it will be shown that for all $\left\{f_{i}\right\}_{i=1}^{n} \in \mathcal{F}_{S}^{n}$,

$$
v^{*}\left(f_{1}, \ldots, f_{n} ; \lambda\right)=\min \left\{\int_{S} \bigvee_{i=1}^{n} p_{i} f_{i} d \lambda:\left(p_{i}\right)_{i=1}^{n} \in \Delta_{n}\right\}
$$

(where $v^{*}$ is as in (2.2)). First observe that

$$
\min _{1 \leq i \leq n} \int_{S} f_{i} \phi_{i} d \lambda=\min \left\{\sum_{i=1}^{n} p_{i} \int_{S} f_{i} \phi_{i} d \lambda:\left(p_{i}\right)_{i=1}^{n} \in \Delta_{n}\right\}
$$

for each $\left\{\phi_{i}\right\}_{i=1}^{n} \in P_{S}$; 
and that

Next calculate

$$
\begin{aligned}
& \sup \left\{\int_{S} \sum_{i=1}^{n} p_{i} f_{i} \phi_{i} d \lambda:\left\{\phi_{i}\right\}_{i=1}^{n} \in \mathcal{P}_{S}\right\} \\
& =\int_{S} \bigvee_{i=1}^{n} p_{i} f_{i} d \lambda \text { for each }\left(p_{i}\right)_{i=1}^{n} \in \Delta_{n} .
\end{aligned}
$$

$$
\begin{aligned}
v^{*}\left(f_{1}, \ldots, f_{n} ; \lambda\right) & =\sup _{\phi \in \mathcal{P}_{S}} \min _{1 \leq i \leq n} \int_{S} f_{i} \phi_{i} d \lambda \\
& =\sup _{\phi \in \mathcal{P}_{S}} \min _{p \in \Delta_{n}} \sum_{i=1}^{n} p_{i} \int_{S} f_{i} \phi_{i} d \lambda \\
& =\min _{p \in \Delta_{n}} \sup _{\phi \in \mathcal{P}_{S}} \int_{S} \sum_{i=1}^{n} p_{i} f_{i} \phi_{i} d \lambda \\
& =\min _{p \in \Delta_{n}} \int_{S} \bigvee_{i=1}^{n} p_{i} f_{i} d \lambda,
\end{aligned}
$$

where the second and fourth equalities in (6.4) follow from (6.2) and (6.3) respectively, and the third equality follows from the minimax theorem of game theory (see, e.g., $\S 6.3$ of $[8]$ ), since $\Delta_{n}$ and $P_{S}$ are compact and convex, and $H(p, \phi):=$ $\sum_{i=1}^{n} p_{i} \int_{S} f_{i} \phi_{i} d \lambda$ is continuous bilinear.

Together, Theorem 1.1 and Proposition 6.1 imply the following result.

THEOREM 6.2. If $\mu_{1}, \ldots, \mu_{n}$ are nonatomic probability measures on $(S, B)$ then

$$
\left\|\bigvee_{i=1}^{n} p_{i} \mu_{i}\right\| \geq(n-M+1)^{-1} \quad \text { for all }\left\{p_{i}\right\}_{i=1}^{n} \text { with } p_{i} \geq 0, \sum_{i=1}^{n} p_{i}=1
$$

One possible interpretation of the conclusion of Theorem 6.2 is this. Suppose the values of the $n$ participants in a cooperative fair-division problem are weighted (by $p_{1}, \ldots, p_{n}$ ) to obtain subprobability values $v_{1}, \ldots, v_{n}$ (where $v_{i}=p_{i} \mu_{i}$ ). The object is then divided optimally, each piece is given to the person who values it most (according to the values $\left\{v_{i}\right\}$ ), and the values of these pieces to the corresponding recipients are then pooled to obtain a "weighted cooperative" value $\left(\left\|\bigvee_{i=1}^{n} p_{i} \mu_{i}\right\|\right)$. Theorem 6.2 then says that no matter what weights are assigned, the weighted cooperative value is at least $(n-M+1)^{-1}$.

The following analog of Proposition 6.1 and Theorem 6.2 for the discrete allocation (matrix theoretic) setting of $\S 5$ is also straightforward.

Proposition 6.3. Let $A=\left(a_{i j}\right)$ be an $n \times k$ stochastic matrix. Then

and

$$
v^{*}(A)=\min \left\{\sum_{j=1}^{k} \max _{1 \leq i \leq n} p_{i} a_{i j}:\left(p_{i}\right)_{i=1}^{n} \in \Delta_{n}\right\}
$$

$$
\sum_{j=1}^{k} \max _{1 \leq i \leq n} p_{i} a_{i j} \geq(n-M+1)^{-1} \quad \text { for all }\left(p_{i}\right)_{i=1}^{n} \in \Delta_{n}
$$

(where $M$ and $v^{*}$ are as in (5.1) and (5.2)). 
Finally, an application of Theorem 1.1 (related to an example of Dvoretzky, Wald, and Wolfowitz [6, §4] is given to statistical decision theory. Suppose there is an $S$-valued (continuous) random variable $X$ which is assumed to have unknown distribution, but which has one of the distributions $\mu_{1}, \ldots, \mu_{n}$. A single observation $X(\omega)$ of $X$ is made, and then it is to be guessed from which of the distributions $\mu_{1}, \ldots, \mu_{n}$ the observation came. A decision rule is simply a (measurable) partition $\left\{S_{i}\right\}_{i=1}^{n}$ of $S$ ("if $X(\omega) \in S_{i}$, then guess distribution $\mu_{i}$ "). A minimax decision rule is a partition which attains the "minimax risk" $R$ given by

$$
\begin{aligned}
R & =R\left(\mu_{1}, \ldots, \mu_{n}\right) \\
& =\inf \left\{\max _{1 \leq i \leq n} P\left(X \notin S_{i} \mid \operatorname{dist}(X)=\mu_{i}\right):\left\{S_{i}\right\}_{i=1}^{n} \in \Pi_{S}\right\} .
\end{aligned}
$$

By (1.2) it follows that

$$
\begin{aligned}
R\left(\mu_{1}, \ldots, \mu_{n}\right) & =\inf \left\{\max _{1 \leq i \leq n}\left(1-\mu_{i}\left(S_{i}\right)\right):\left\{S_{i}\right\}_{i=1}^{n} \in \Pi_{S}\right\} \\
& =1-v^{*}\left(\mu_{1}, \ldots, \mu_{n}\right) .
\end{aligned}
$$

Theorem 1.1 and (6.6) together imply the following result.

THEOREM 6.4. If $\mu_{1}, \ldots, \mu_{n}$ are nonatomic probability measures on $(S, B)$, then the minimax risk $R$ satisfies

$$
n^{-1}(n-M) \leq R\left(\mu_{1}, \ldots, \mu_{n}\right) \leq(n-M+1)^{-1}(n-M),
$$

where $M$ is as in (1.1). Moreover, both bounds are best possible for the functional $M$.

\section{REFERENCES}

1. R. Ash, Real analysis and probability, Academic Press, New York, 1972.

2. S. Demko and T. Hill, Equitable distribution of indivisible objects, preprint, 1985.

3. L. Dor, On projections in $L_{1}$, Ann. of Math (2)102 (1975), 463-474.

4. L. Dubins and E. Spanier, How to cut a cake fairly, Amer. Math. Monthly 68 (1961), 1-17.

5. N. Dunford and J. Schwartz, Linear operators, Part I, Interscience, New York, 1958.

6. A. Dvoretzky, A. Wald and J. Wolfowitz, Relations among certain ranges of vector measures, Pacific J. Math. 1 (1951), 59-74.

7. T. Hill, Equipartitioning the common domain of nonatomic measures, Math. Z. 189 (1985), 415-419.

8. J. Stoer and C. Witzgall, Convexity and optimization in finite dimensions. I, Grundlehren Math. Wiss., Band 163, Springer-Verlag, New York, 1970.

9. K. Urbanik, Quelques théorèmes sur les mesures, Fund. Math. 41 (1955), 150-162.

School of Mathematics, Georgia Institute of Technology, Atlanta, GeorGIA 30332 